$\underline{\text { Preprint typeset in JHEP style - HYPER VERSION }}$

LPTA/09-035, IPhT-T09/066

\title{
Quantum mirror symmetry and twistors
}

\author{
Sergei Alexandrov ${ }^{1}$, Frank Saueressig ${ }^{2}$ \\ 1 Laboratoire de Physique Théorique \&3 Astroparticules, CNRS UMR 520\%, \\ Université Montpellier II, 34095 Montpellier Cedex 05, France \\ 2 Institut de Physique Théorique, CEA, IPhT, F-91191 Gif-sur-Yvette, France \\ CNRS, URA 2306, F-91191 Gif-sur-Yvette, France
}

\begin{abstract}
Using the twistor approach to hypermultiplet moduli spaces, we derive the worldsheet, $\mathrm{D}(-1)$, and D1-instanton contributions to the generalized mirror map, relating Type IIA and Type IIB string theory compactified on generic mirror CalabiYau threefolds. For this purpose, we provide a novel description of the twistor space underlying the Type IIB hypermultiplet moduli space where the $\operatorname{SL}(2, \mathbb{Z})$-action is found to be free from quantum corrections. The extent to which instanton effects may resolve the perturbative singularities of the moduli space metric is discussed.
\end{abstract}




\section{Contents}

1. Introduction 1

2. Twistor description of toric quaternion-Kähler spaces 5

3. Twistor description of perturbative HM moduli spaces 8

3.1 Type IIA compactified on a CY threefold $X$

3.2 Type IIB compactified on a CY threefold $Y$

4. Instanton corrected Type IIB HM moduli space 12

5. The non-perturbative mirror map 16

6. Perturbative singularities and instanton corrections 18

6.1 The perturbative one-loop singularity

6.2 The effect of D-instanton corrections 20

7. Discussion and outlook 22

A. Type IIA and Type IIB twistor spaces and their relation 23

A.1 Instanton corrected Type IIA HM moduli space

A.2 Mirror symmetry of the twistor spaces 26

B. Poisson resummation of the twistor lines 30

B.1 Resuming $\mathcal{I}_{\gamma}^{(\nu)}(\mathbf{z})$

B.2 Resuming the Type IIA twistor lines

Q. SL $(2, \mathbb{Z})$-transformation of the Type IIB twistor lines 36

\section{Introduction}

Instanton effects are one of the key ingredients in understanding the mathematical structures of string theory at the non-perturbative level. They dominate the dynamics at strong coupling and play a prominent role in the non-perturbative dualities which 
are vital for exploring in this regime. Moreover, there is evidence that they are capable of resolving the singularities appearing in the perturbative analysis of the string theory moduli space [四].

An important laboratory, where such effects can be studied in detail, is the compactification of Type II strings on a Calabi-Yau threefold (CY). In this case the lowenergy effective action (LEEA) has $N=2$ supersymmetry and receives space-time instanton corrections from Euclidean D-branes and NS5-branes wrapping supersymmetric cycles of the internal space [2]. Supersymmetry then dictates that the LEEA is characterized by two manifolds: a special Kähler (SK) space $\mathcal{K}$ determining its vector multiplet sector, and a quaternion-Kähler (QK) space $\mathcal{M}$ underlying its hypermultiplet sector. For Type IIA strings compactified on a CY $X$ and Type IIB strings on $Y, \mathcal{K}$ is given by the Kähler moduli space $\mathcal{K}_{K}(X)$ and the moduli space of complex structures $\mathcal{K}_{C}(Y)$, respectively:

\begin{tabular}{|c|c|c|}
\hline & Type IIA $/ X$ & Type IIB $/ Y$ \\
\hline $\begin{array}{c}\text { moduli } \\
\text { space }\end{array}$ & $\mathcal{K}_{K}(X) \times \mathcal{M}_{C}(X)$ & $\mathcal{K}_{C}(Y) \times \mathcal{M}_{K}(Y)$ \\
\hline
\end{tabular}

At string tree-level, $\mathcal{M}_{C}(X)$ and $\mathcal{M}_{K}(Y)$ can be obtained from $\mathcal{K}_{C}(X)$ and $\mathcal{K}_{K}(Y)$ via

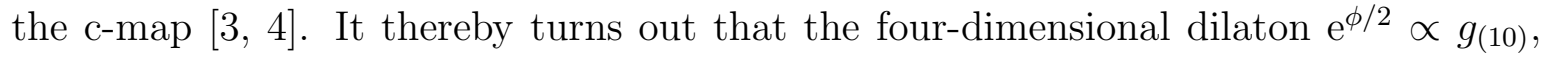
proportional to the ten-dimensional string coupling constant, enters into $\mathcal{M}$. Thus both hypermultiplet sectors are subject to perturbative and non-perturbative string corrections.

A striking feature of these moduli spaces is their conjectured relation via mirror symmetry [5]. In its "generalized" or "non-perturbative" formulation, this duality states that the moduli spaces arising from the compactification of Type IIA strings on $X$ and Type IIB strings on $\tilde{X}$ (mirror to $X$ ) are actually identical. On the vector multiplet side this amounts to the well-supported classical mirror symmetry [6], which equates the complex structure moduli space of $X$ with the complexified Kähler moduli space of $\tilde{X}, \mathcal{K}_{C}(X)=\mathcal{K}_{K}(\tilde{X})$. Generalized mirror symmetry further implies the identification

$$
\mathcal{M}_{C}(X)=\mathcal{M}_{K}(\tilde{X}),
$$

which is supposed to hold upon including the non-perturbative $g_{s}$-corrections originating from D- and NS5-brane instantons [2, [7]. In mathematical terms (11.1) translates into an equivalence between elements of the derived category $\mathcal{D}(\tilde{X})$ of coherent sheaves (related to $\mathcal{M}_{K}(\tilde{X})$ ) and elements in the derived Fukaya category $\mathcal{F}(X)$ of SLAG submanifolds (related to $\mathcal{M}_{C}(X)$ ) (see [8] for an introduction). In particular, it encompasses the homological mirror symmetry conjecture [9]. 
At the level of the LEEA, mirror symmetry is realized via the generalized (or nonperturbative) mirror map which relates the physical fields of the Type IIA compactification, parameterizing $\mathcal{M}_{C}(X)$, to the physical fields of the Type IIB compactification, providing coordinates on $\mathcal{M}_{K}(\tilde{X})$. The classical limit of this map has been obtained in [10]. Since the moduli spaces receive quantum corrections from both worldsheet and D-brane instantons, it is expected, however, that the full generalized mirror map will also be subject to such corrections. The main result of this paper, which can be found in eq. (5.2), is the explicit construction of this map, including the quantum corrections from worldsheet, $\mathrm{D}(-1)$, and D1-instantons.

The derivation of the generalized mirror map requires the detailed knowledge of the perturbative and non-perturbative corrections to $\mathcal{M}$. For the compactifications at hand these are given by perturbative worldsheet and $g_{s}$-corrections, contributions from D2branes wrapping the SLAG three-cycles in Type IIA mirror to odd branes $(\mathrm{D}(-1), \mathrm{D} 1$, D3, D5) wrapping complex cycles of $\tilde{X}$ in Type IIB [11, and NS5-branes. A fruitful route for determining these corrections explicitly is via a chain of non-perturbative symmetries and dualities $[12$, 13]. A key role is played by the $\operatorname{SL}(2, \mathbb{Z})$ duality of the Type IIB string [10] and the symplectic covariance of the Type IIA theory [14], which are believed to hold also non-perturbatively. ${ }^{1}$ At the classical level, the actions of these dualities on the physical fields parameterizing the moduli spaces can be deduced from the dimensional reduction of the ten-dimensional supergravity action. At the quantum level, we then define the "physical fields" coordinatizing $\mathcal{M}$ by the requirement that they obey the classical transformation laws with respect to $\operatorname{SL}(2, \mathbb{Z})$ on the Type IIB side and the symplectic group on the Type IIA side, respectively. Thus, by definition, the action of these transformations on $\mathcal{M}$ does not receive quantum corrections.

Following this route, [16] obtained exact results for the $\mathrm{D}(-1)$ and D1-instanton corrections by implementing the $\mathrm{SL}(2, \mathbb{Z})$-invariance of the Type IIB string. (For a recent rederivation of some of these results from a topological string perspective see [17.) Using the classical limit of the mirror map together with the assumption that the instanton numbers on the Type IIA and Type IIB side agree, this result also yields the mirror symmetric "A-type" D2-instanton contributions [18, 12, 13]. Subsequently, these corrections have been generalized to include all D2-instantons [19, 20] and there are also partial results on NS5-brane instantons [21, 22]. Determining the exact contribution of the latter will, however, require detailed knowledge of the generalized mirror map, which provides a strong motivation for our investigation.

Our work heavily draws on twistor space description of QK spaces [23, 24]. This approach encodes the complicated quaternion-Kähler geometry of $\mathcal{M}$ in a set of simple

\footnotetext{
${ }^{1}$ For a recent analysis of the interplay between these symmetries and wall-crossing phenomena see 15.
} 
holomorphic functions appearing as transition functions between locally flat Darboux patches of the twistor space $\mathcal{Z}$ of $\mathcal{M}$. These transition functions depend on the complex coordinates on the twistor space, the so-called twistor lines, and play a similar role as the holomorphic prepotential for the special Kähler spaces. In this framework, the problem of determining the instanton corrections to $\mathcal{M}$ is equivalent to finding appropriate holomorphic functions which respect the non-perturbative symmetries of the theory.

The strategy for constructing the generalized mirror map is then to lift the nonperturbative duality transformations from $\mathcal{M}$ to $\mathcal{Z}$, where they induce transformations of the twistor lines. The twistor lines then transform in a particular representation of the duality group. At the classical level, the actions of $\operatorname{SL}(2, \mathbb{Z}) S$-duality and electricmagnetic rotations have already been obtained in [19]. We will show that, locally, these classical transformations remain uncorrected upon including D-instanton contributions. Imposing compatibility between the transformation laws of the physical fields and the twistor lines then allows us to determine the instanton corrections to the generalized mirror map. At the classical level, this computation has already been carried out in [19] recovering the result of [10].

Motivated by the observation that D1-instantons play an essential role in smoothing the conifold singularity appearing in the perturbative hypermultiplet metric [1, 18], we also investigate the effect of the D-instanton corrections on the perturbative (1loop) singularity of $\mathcal{M}$ [25, 26]. In this context, we observe that the four-dimensional string coupling (defined via the contact potential, see eq. (2.2) below) does not become strongly coupled, while the moduli space metric contains a curvature singularity at a finite value of the ten-dimensional string coupling. The latter is not resolved by the instanton corrections considered here, and we conjecture that the resolution of this singularity will require the inclusion of NS5-branes.

The rest of the paper is organized as follows. In Sections 2 and 3 we review the general twistor space construction of toric quaternion-Kähler manifolds and its application to the perturbative hypermultiplet moduli spaces arising in Type IIA and Type IIB string compactifications, respectively. Section 4 contains our new description of the instanton corrected Type IIB twistor space. In Appendices A and B we show that this formulation is gauge-equivalent to the Type IIA description [19, establishing mirror symmetry at the level of the twistor spaces. This result is used to derive the $\mathrm{SL}(2, \mathbb{Z})$-transformation of the instanton corrections to the twistor lines in the Type IIB framework and the non-perturbative mirror map in Section 5. Section 6 contains our analysis of the perturbative singularities of $\mathcal{M}$. A brief discussion of our results together with an outlook is given in Section 7 . Finally, the technical details about the $\operatorname{SL}(2, \mathbb{Z})$-transformations of the Type IIB twistor lines can be found in Appendix C. 


\section{Twistor description of toric quaternion-Kähler spaces}

In this section we summarize the twistor space description of (toric) QK manifolds recently developed in [24]. It is based on the Lebrun-Salamon theorem [27, 28, 24], which guarantees that the metric on $\mathcal{M}$ can be recovered from the complex contact structure $^{2}$ on its twistor space $\mathcal{Z}$. Moving from $\mathcal{M}$ to $\mathcal{Z}$ brings the advantage that all the geometrical data can be encoded in a set of holomorphic transition functions which relate the contact structure in different patches of $\mathcal{Z}$. These functions provide an invaluable tool when studying deformations of QK spaces. In the following, we will review these geometric objects and their relation to the metric on $\mathcal{M}$, which will be central in our discussion of instanton corrections to HM moduli spaces.

A QK manifold $\mathcal{M}$ is a real $4 d$-dimensional Riemannian manifold with holonomy contained in $U S p(d) \times S U(2)$. Locally it admits a triplet of almost complex structures which satisfy the algebra of unit quaternions. The holonomy constraint implies that $\mathcal{M}$ carries a canonical $\mathrm{SU}(2)$-connection $\vec{p}$ with components $p_{-}=\left(p_{+}\right)^{*}, p_{3}=\left(p_{3}\right)^{*}$, which is given by the $\mathrm{SU}(2)$-part of the Levi-Civita connection. We call $\mathcal{M}$ toric, if it admits $d+1$ commuting isometries.

The twistor space $\mathcal{Z}$ is a $\mathbb{C} P^{1}$-bundle over $\mathcal{M}$, i.e., $\mathcal{Z}=\mathcal{M} \times \mathbb{C} P^{1}$ locally, and we use local coordinates $x^{\mu}$ on $\mathcal{M}$ and the complex coordinate $\mathbf{z}$ on $\mathbb{C} P^{1}$, respectively. $\mathcal{Z}$ is Kähler-Einstein and its connection is given by $\vec{p}$. Furthermore, it carries a real structure $\tau$ which acts as the antipodal map on $\mathbb{C} P^{1}$ and leaves the coordinates of $\mathcal{M}$ invariant, $\tau:\left\{x^{\mu}, \mathbf{z}\right\} \mapsto\left\{x^{\mu},-\overline{\mathbf{z}}^{-1}\right\}$.

The key ingredient in the construction is the complex contact structure of $\mathcal{Z}$. On the open covering $\hat{\mathcal{U}}_{i}$ of $\mathcal{Z}$, the latter can be represented by a set of holomorphic oneforms $\mathcal{X}^{[i]}$, such that the holomorphic top form

$$
\kappa^{[i]}=\mathcal{X}^{[i]} \wedge\left(\mathrm{d} \mathcal{X}^{[i]}\right)^{d} \neq 0
$$

is nowhere vanishing. On each patch,

$$
\mathcal{X}^{[i]}=2 \mathrm{e}^{\Phi_{[i]}} \frac{D \mathbf{z}}{\mathbf{z}}
$$

is proportional to the canonical $(1,0)$ form $D \mathbf{z}=\mathrm{d} \mathbf{z}+p_{+}-\mathrm{i} p_{3} \mathbf{z}+p_{-} \mathbf{z}^{2}$ and subject to the reality constraint $\overline{\tau(\mathcal{X}[i])}=-\mathcal{X}^{[i]}$ where we assume that $\tau$ maps the patch $\hat{\mathcal{U}}_{i}$ onto $\hat{\mathcal{U}}_{\bar{\imath}}$, which can always be achieved through a refinement of the covering. The "contact potential" $\Phi_{[i]} \equiv \Phi_{[i]}\left(x^{\mu}, \mathbf{z}\right)$ is a function on $\hat{\mathcal{U}}_{i} \subset \mathcal{Z}$. It is holomorphic along the $\mathbb{C} P^{1}$ fiber, defined up to an additive holomorphic function on $\hat{\mathcal{U}}_{i}$, and chosen such that the

\footnotetext{
${ }^{2}$ For a general introduction to contact geometry, see [29].
} 
right-hand side of (2.2) is a holomorphic (i.e. $\bar{\partial}$-closed) one-form. Furthermore, the reality constraint on $\mathcal{X}^{[i]}$ implies that $\overline{\tau\left(\Phi_{[i]}\right)}=\Phi_{[\bar{i}}$.

By a variation of Darboux's theorem, for an appropriate open covering, in each patch $\hat{\mathcal{U}}_{i}$ one can find complex coordinates $\xi_{[i]}^{\Lambda}, \tilde{\xi}_{\Lambda}^{[i]}, \alpha^{[i]}(\Lambda=0, \ldots, d-1)$ such that the $\mathcal{X}^{[i]}$ takes the canonical form

$$
\mathcal{X}^{[i]} \equiv \mathrm{d} \alpha^{[i]}+\xi_{[i]}^{\Lambda} \mathrm{d} \tilde{\xi}_{\Lambda}^{[i]} .
$$

We choose these coordinates to satisfy

$$
\overline{\tau\left(\xi_{[i]}^{\Lambda}\right)}=\xi_{[\bar{l}]}^{\Lambda}, \quad \overline{\tau\left(\tilde{\xi}_{\Lambda}^{[i]}\right)}=-\tilde{\xi}_{\Lambda}^{[\bar{l}]}, \quad \overline{\tau\left(\alpha^{[i]}\right)}=-\alpha^{[\bar{l}]} .
$$

The global structure of $\mathcal{Z}$ can then be encoded in a set of holomorphic transition functions relating the sets of coordinates on the overlap of two patches $\hat{\mathcal{U}}_{i} \cap \hat{\mathcal{U}}_{j}$, thereby preserving (2.3). Together with the $d+1$ additional real numbers $c_{\Lambda}, c_{\alpha}$, called the "anomalous dimensions", these transition functions contain all the geometric information of the twistor space and the corresponding QK base.

Extracting the metric on $\mathcal{M}$ requires the construction of the twistor lines of $\mathcal{Z}$ by expressing the complex coordinates $\xi_{[i]}^{\Lambda}, \tilde{\xi}_{\Lambda}^{[i]}, \alpha^{[i]}$ in terms of the coordinates $\left\{x^{\mu}, \mathbf{z}\right\}$. For a generic QK metric, where the transition functions depend on all coordinates $\xi_{[i]}^{\Lambda}, \tilde{\xi}_{\Lambda}^{[i]}, \alpha^{[i]}$, this is fairly difficult. For the purpose of this paper it suffices, however, to consider the case where $\mathcal{M}$ is toric. In this case the moment maps associated to the $d+1$ isometries [30] provide independent global $\mathcal{O}(2)$ sections on $\mathcal{Z}$, which can be taken to be the complex coordinates $\xi_{[i]}^{\Lambda}$ and the unit function. The resulting twistor lines $\xi_{[i]}^{\Lambda}$ are globally defined and take the form

$$
\xi^{\Lambda} \equiv \xi_{[i]}^{\Lambda}=Y^{\Lambda} \mathbf{z}^{-1}+A^{\Lambda}-\bar{Y}^{\Lambda} \mathbf{z}
$$

on all patches $\hat{\mathcal{U}}_{i}$. In fact, one can choose $Y^{0} \equiv \mathcal{R}$ to be real by fixing the $U(1)$-action corresponding to phase rotations of $\mathbf{z}$. Together with the $d+1$ additional real coordinates $\left\{B_{\Lambda}, B_{\alpha}\right\}$ introduced in (2.9), $\mathcal{R}, Y^{a}, \bar{Y}^{a}, A^{\Lambda}$ provide a convenient coordinate system on $\mathcal{M}$.

As a consequence of (2.5), the complex coordinates $\xi^{\Lambda}, \tilde{\xi}_{\Lambda}$ and $\alpha$ must now be related by transition functions which preserve $\xi^{\Lambda}$ and the unit function. The general form of such contact transformations is given by

$$
\tilde{\xi}_{\Lambda}^{[i]}=\tilde{\xi}_{\Lambda}^{[j]}-\partial_{\xi^{\Lambda}} H^{[i j]}, \quad \alpha^{[i]}=\alpha^{[j]}-H^{[i j]}+\xi^{\Lambda} \partial_{\xi^{\Lambda}} H^{[i j]},
$$

where the transition functions $H^{[i j]}(\xi)$ are independent of $\tilde{\xi}_{\Lambda}^{[i]}, \alpha^{[i]}$. The reality condition (2.4) and the consistency conditions appearing on the overlap of three patches furthermore imply the additional constraints

$$
\overline{\tau\left(H^{[i j]}\right)}=-H^{[\bar{\imath}]}, \quad H^{[i j]}+H^{[j k]}=H^{[i k]} .
$$


Besides, it is important to note that the transition functions $H^{[i j]}$ do not specify the twistor space uniquely, but are subject to the gauge equivalence

$$
H^{[i j]} \mapsto H^{[i j]}+T^{[i]}-T^{[j]}
$$

where $T^{[i]}\left(\xi^{\Lambda}\right)$ are holomorphic functions regular in the patch $\hat{\mathcal{U}}_{i}$. Essentially they capture the possibility to perform a local change of coordinates in $\hat{\mathcal{U}}_{i}$, which leaves the contact form (2.3) invariant. We shall often abuse notation and define $H^{[i j]}$ away from the overlap $\hat{\mathcal{U}}_{i} \cap \hat{\mathcal{U}}_{j}$ (in particular when the two patches do not intersect) using analytic continuation and the second equation in (2.7) to interpolate from $\hat{\mathcal{U}}_{i}$ to $\hat{\mathcal{U}}_{j}$. Ambiguities in the choice of path can be dealt with on a case by case basis.

The gluing conditions (2.6) together with the requirement that the contact form takes the form (2.2) are sufficient to determine the twistor lines $\tilde{\xi}_{\Lambda}^{[i]}, \alpha^{[i]}$ for the toric case [24]: ${ }^{3}$

$$
\begin{aligned}
\tilde{\xi}_{\Lambda}^{[i]} & =\frac{\mathrm{i}}{2} B_{\Lambda}+\frac{1}{2} \sum_{j} \oint_{C_{j}} \frac{\mathrm{d} \mathbf{z}^{\prime}}{2 \pi \mathrm{i} \mathbf{z}^{\prime}} \frac{\mathbf{z}^{\prime}+\mathbf{z}}{\mathbf{z}^{\prime}-\mathbf{z}} \partial_{\xi^{\Lambda}} H^{[i j]}\left(\xi\left(\mathbf{z}^{\prime}\right)\right)+c_{\Lambda} \log \mathbf{z}, \\
\alpha^{[i]} & =\frac{\mathrm{i}}{2} B_{\alpha}+\frac{1}{2} \sum_{j} \oint_{C_{j}} \frac{\mathrm{d} \mathbf{z}^{\prime}}{2 \pi \mathrm{i} \mathbf{z}^{\prime}} \frac{\mathbf{z}^{\prime}+\mathbf{z}}{\mathbf{z}^{\prime}-\mathbf{z}}\left[H-\xi^{\Lambda} \partial_{\xi^{\Lambda}} H\right]^{[i j]}+c_{\alpha} \log \mathbf{z}+c_{\Lambda}\left(Y^{\Lambda} \mathbf{z}^{-1}+\bar{Y}^{\Lambda} \mathbf{z}\right) .
\end{aligned}
$$

Here, $\mathbf{z} \in \mathcal{U}_{i}$, with $\mathcal{U}_{i}$ denoting the projection of $\hat{\mathcal{U}}_{i}$ to $\mathbb{C} P^{1}$, and $C_{j}$ is a contour surrounding $\mathcal{U}_{j}$. The "integration constants" $B_{\Lambda}, B_{\alpha}$ provide the extra $d+1$ coordinates on $\mathcal{M}$ mentioned above. Note that the twistor lines are not regular in the patches around $\mathbf{z}=0$ and $\mathbf{z}=\infty$ but may contain singular terms. These terms are weighted by the anomalous dimensions $c_{\Lambda}, c_{\alpha}$ which control the singular behavior at these points. These are the only singularities admissible by regular metrics. Moreover, the contact potential turns out to be independent of $\mathbf{z}$ and the same in all patches. Explicitly it is given by

$$
e^{\Phi}=\frac{1}{4} \sum_{j} \oint_{C_{j}} \frac{\mathrm{d} \mathbf{z}^{\prime}}{2 \pi \mathrm{i} \mathbf{z}^{\prime}}\left(\mathbf{z}^{\prime-1} Y^{\Lambda}-\mathbf{z}^{\prime} \bar{Y}^{\Lambda}\right) \partial_{\xi^{\Lambda}} H^{[i j]}\left(\xi\left(\mathbf{z}^{\prime}\right)\right)+\frac{1}{2}\left(c_{\Lambda} A^{\Lambda}+c_{\alpha}\right)
$$

Note that due to consistency conditions (2.7), the index $i$ of the transition functions in (2.9), (2.10) can be replaced by any other patch index without affecting the result.

Given the twistor lines (2.5) and (2.9), the QK metric on $\mathcal{M}$ can be obtained using the general formalism [24], which considerably simplifies for the toric case. This formalism utilizes the Laurent expansion of the twistor lines in the patch $\hat{\mathcal{U}}_{+}$around

\footnotetext{
${ }^{3}$ Formulas for the twistor lines arising from an infinitesimal deformation away from the toric case have been obtained in [24].
} 
$\mathbf{z}=0$. Substituting this expansion into the contact one-form (2.3) and comparing to (2.2) allows to determine the $\mathrm{SU}(2)$ connection $\vec{p}$ and the contact potential in terms of the Laurent coefficients of this expansion. The SU(2) connection then gives the triplet of quaternionic forms $\vec{\omega}_{\mathcal{M}}$, and in particular

$$
\omega_{\mathcal{M}, 3}=\mathrm{d} p_{3}+2 \mathrm{i} p_{+} \wedge p_{-}
$$

Upon determining the almost complex structure $J_{3}$, the metric then follows from $g_{\mathcal{M}}\left(J_{3} X, Y\right)=\omega_{\mathcal{M}, 3}(X, Y)$. The former can be specified through a basis of local oneforms on $\mathcal{M}$ of Dolbeault type $(1,0)$ with respect to $J_{3}$. Let us denote by $\tilde{\xi}_{\Lambda, 0}^{[+]}$and $\alpha_{0}^{[+]}$ the constant terms in the Laurent expansion of $\tilde{\xi}_{\Lambda}^{[+]}$and $\alpha^{[+]}$, respectively. Then by expanding the holomorphic one-forms $\mathrm{d} \xi^{\Lambda}, \mathrm{d} \tilde{\xi}_{\Lambda}^{[+]}$, and $\mathrm{d} \alpha^{[+]}$around $\mathbf{z}=0$ and projecting the result along the base $\mathcal{M}$, it can be shown that a suitable basis is given by ${ }^{4}$

$$
\Pi^{a}=\mathrm{d}\left(Y^{a} / \mathcal{R}\right), \quad \tilde{\Pi}_{\Lambda}=\mathrm{d} \tilde{\xi}_{\Lambda, 0}^{[+]}+c_{\Lambda} \mathrm{d} \log \mathcal{R}, \quad \tilde{\Pi}_{\alpha}=-\mathrm{d} \alpha_{0}^{[+]}+c_{\Lambda} \mathrm{d} A^{\Lambda}-c_{\alpha} \mathrm{d} \log \mathcal{R}
$$

where $a$ runs over $1, \ldots, d-1$. These one-forms will play a central role in our discussion of the regularity of the metric on $\mathcal{M}$ in Section 6 . In this context, it is often convenient to trade the coordinate $\mathcal{R}$ for the variable $\mathrm{e}^{\Phi}$. As we shall see below, this is natural for the hypermultiplet moduli space, since the contact potential $\mathrm{e}^{\Phi}$ is identified with the four-dimensional dilaton $\mathrm{e}^{\phi}$.

\section{Twistor description of perturbative HM moduli spaces}

We now review the twistor space description of the perturbative hypermultiplet moduli spaces arising from compactifying Type II string theory on a CY threefold. This will provide our starting point for determining the instanton corrections to the classical mirror map in Section 5. Owed to the gauge-invariance of the $p$-forms appearing in the original ten-dimensional action before compactification, the perturbative hypermultiplet moduli spaces admit a Heisenberg group of isometries and therefore fall into the class of toric QK manifolds discussed in the previous section.

\subsection{Type IIA compactified on a CY threefold $X$}

The hypermultiplet moduli space $\mathcal{M}_{C}(X)$ in Type IIA string theory compactified on a CY threefold $X$ is a QK manifold of quaternionic dimension $d=h^{2,1}(X)+1$ [3], 4, 31]. It describes the dynamics of the complex structure moduli $X^{\Lambda}=\int_{\gamma^{\Lambda}} \Omega, F_{\Lambda}=\int_{\gamma_{\Lambda}} \Omega$, the

\footnotetext{
${ }^{4} \mathrm{Up}$ to overall factors, these correspond to the $(1,0)$-forms $\Pi^{a}, \tilde{\Pi}_{I}$ introduced in [24], which are further simplified by utilizing that $p_{+}$itself is of Dolbeault-type $(1,0)$.
} 
RR scalars $\zeta^{\Lambda}, \tilde{\zeta}_{\Lambda}$ originating as similar integrals of the RR 3-form, the four-dimensional dilaton $\mathrm{e}^{\phi}=1 / g_{(4)}^{2}$ and the Neveu-Schwarz (NS) axion $\sigma$, dual to the NS two-form $B$ in four dimensions. Here, $\left\{\gamma^{\Lambda}, \gamma_{\Lambda}\right\}$ represent a symplectic basis of A- and B-cycles in $H_{3}(X, \mathbb{Z})$ with intersection product $\left\langle\gamma^{\Lambda}, \gamma_{\Sigma}\right\rangle=\delta_{\Sigma}^{\Lambda}$. The sets $\left\{X^{\Lambda}, F_{\Lambda}(X)\right\}$ and $\left\{\zeta^{\Lambda}, \tilde{\zeta}_{\Lambda}\right\}$ transform as symplectic vectors with respect to electric-magnetic duality $\operatorname{Sp}(2 d, \mathbb{Z})$. The $X^{\Lambda}$ provide a set of homogeneous coordinates on the space of complex structure deformations $\mathcal{K}_{C}(X)$, and (away from the vanishing locus of $X^{0}$ ) may be traded for the inhomogeneous coordinates $z^{a}=X^{a} / X^{0}$.

At string tree level, $\mathcal{M}_{C}(X)$ can be obtained from the special Kähler space $\mathcal{K}_{C}(X)$ (describing the vector multiplet moduli space of Type IIB strings compactified on the same CY $X$ ) by the $c$-map [3, 4. The latter space is completely characterized by the prepotential $F\left(X^{\Lambda}\right)$, a homogeneous function of degree two of the A-type periods $X^{\Lambda}$, such that the B-type periods are given by $F_{\Lambda}=\partial F / \partial X^{\Lambda}$. Therefore, the same is true for the tree level HM moduli space. At one-loop, the metric on $\mathcal{M}_{C}(X)$ receives a correction proportional to the Euler class $\chi_{X}=2\left(h^{1,1}(X)-h^{2,1}(X)\right)$. Based on the string theory amplitudes 32, 33], the one-loop corrected QK metric was calculated in [25]. It is believed to be the correct metric on $\mathcal{M}_{C}(X)$ to all orders in perturbation theory [33, 25, 26, 24].

The twistor space formulation of $\mathcal{M}_{C}(X)$ was worked out in [24]. As illustrated in the left diagram of Fig. 2, it utilizes two patches $\hat{\mathcal{U}}_{+}, \hat{\mathcal{U}}_{-}$which project to open disks centered around $\mathbf{z}=0$ and $\mathbf{z}=\infty$ on $\mathbb{C} P^{1}$, and a third patch $\hat{\mathcal{U}}_{0}$ which projects to the rest of $\mathbb{C} P^{1}$. The transition functions between these patches and the anomalous dimensions are given by

$$
H^{[0+]}=-\frac{\mathrm{i}}{2} F\left(\xi^{\Lambda}\right), \quad H^{[0-]}=-\frac{\mathrm{i}}{2} \bar{F}\left(\xi^{\Lambda}\right), \quad c_{\alpha}=\frac{\chi_{X}}{96 \pi},
$$

with $c_{\Lambda}=0$. The non-vanishing $c_{\alpha}$ incorporates the one-loop correction.

The twistor lines arising from (3.1) are readily computed from (2.9). Motivated by symplectic invariance, it is convenient to express the twistor line $\alpha^{[i]}$ in terms of

$$
\alpha_{\mathrm{A}}^{[i]} \equiv 4 \mathrm{i} \alpha^{[i]}+2 \mathrm{i} \tilde{\xi}_{\Lambda}^{[i]} \xi^{\Lambda}
$$

so that the symplectic form (2.3) becomes

$$
\mathcal{X}^{[i]}=\frac{1}{4 \mathrm{i}} \mathrm{d} \alpha_{\mathrm{A}}^{[i]}+\frac{1}{2}\left(\xi^{\Lambda} \mathrm{d} \tilde{\xi}_{\Lambda}^{[i]}-\tilde{\xi}_{\Lambda}^{[i]} \mathrm{d} \xi^{\Lambda}\right) .
$$

The twistor lines in the patch $\hat{\mathcal{U}}_{0}$ resulting from (3.1) are then given by 34, 24]

$$
\begin{aligned}
\xi^{\Lambda} & =\zeta^{\Lambda}+\mathcal{R}\left(\mathbf{z}^{-1} z^{\Lambda}-\mathbf{z} \bar{z}^{\Lambda}\right) \\
-2 \mathrm{i} \tilde{\xi}_{\Lambda}^{[0]} & =\tilde{\zeta}_{\Lambda}+\mathcal{R}\left(\mathbf{z}^{-1} F_{\Lambda}(z)-\mathbf{z} \bar{F}_{\Lambda}(\bar{z})\right) \\
\alpha_{\mathrm{A}}^{[0]} & =\sigma+\mathcal{R}\left(\mathbf{z}^{-1} W(z)-\mathbf{z} \bar{W}(\bar{z})\right)+\frac{\mathrm{i} \chi x}{24 \pi} \log \mathbf{z} .
\end{aligned}
$$


Here we set

$$
W(z) \equiv F_{\Lambda}(z) \zeta^{\Lambda}-z^{\Lambda} \tilde{\zeta}_{\Lambda}
$$

and used the relation between the generic coordinates $Y^{a}, A^{\Lambda}, B_{\Lambda}, B_{\alpha}$ introduced in the previous section and the physical Type IIA fields

$$
\zeta^{\Lambda}=A^{\Lambda}, \quad \tilde{\zeta}_{\Lambda}=B_{\Lambda}+A^{\Sigma} \operatorname{Re} F_{\Lambda \Sigma}(z), \quad \sigma=-2 B_{\alpha}-A^{\Lambda} B_{\Lambda}, \quad Y^{a}=\mathcal{R} z^{a}
$$

The contact potential resulting from (3.1) is given by

$$
\mathrm{e}^{\Phi_{\text {pert }}}=\frac{\mathcal{R}^{2}}{4} K(z, \bar{z})+\frac{\chi_{X}}{192 \pi}
$$

where $K(z, \bar{z}) \equiv-2 \operatorname{Im}\left(\bar{z}^{\Lambda} F_{\Lambda}\right)$. This Type IIA twistor space formulation is adapted to the symplectic covariance of the theory. Indeed, $\left\{\xi^{\Lambda},-2 \mathrm{i} \tilde{\xi}_{\Lambda}^{[0]}\right\}$ transforms as a symplectic vector while $\alpha_{\mathrm{A}}^{[0]}$ and $\mathrm{e}^{\Phi_{\text {pert }}}$ are symplectic invariants. Thus the contact form (3.3) is also invariant, making the symplectic covariance manifest. This completes the twistor description of the perturbative Type IIA HM moduli space.

\subsection{Type IIB compactified on a CY threefold $Y$}

The QK manifold $\mathcal{M}_{K}(Y)$ arising from compactifying Type IIB string theory on a CY threefold $Y$ has quaternionic dimension $d=h^{1,1}(Y)+1$. It describes the dynamics of the Kähler moduli $z^{a} \equiv b^{a}+\mathrm{i} t^{a}=\int_{\gamma^{a}} \mathcal{J}$, the RR scalars $c^{0}, c^{a}, c_{a}, c_{0}$ obtained by integrating appropriate combinations of RR forms along even dimensional cycles, the four-dimensional dilaton $\mathrm{e}^{\phi}$ and the NS axion $\psi$. Here $\mathcal{J} \equiv B+\mathrm{i} J=z^{a} \omega_{a}$ is the complexified Kähler form on $Y$. Furthermore, $\gamma^{a}, a=1, \ldots, h^{1,1}(Y)$, form a basis of 2-cycles (Poincaré dual to 4-forms $\omega^{a}$ ), $\gamma_{a}$ a basis of 4-cycles (Poincaré dual to 2-forms $\left.\omega_{a}\right)$, and $\kappa_{a b c}=\int_{Y} \omega_{a} \omega_{b} \omega_{c}=\left\langle\gamma_{a}, \gamma_{b}, \gamma_{c}\right\rangle$ is the triple intersection product in $H_{4}(Y, \mathbb{Z})$. In the large volume limit, the four-dimensional dilaton $\phi$ is related to the ten-dimensional string coupling $g_{(10)}$ via $\mathrm{e}^{\phi}=\frac{1}{2} V\left(t^{a}\right) /\left(g_{(10)}\right)^{2}$, where $V\left(t^{a}\right)=\frac{1}{6} \int_{Y} J \wedge J \wedge J=\frac{1}{6} \kappa_{a b c} t^{a} t^{b} t^{c}$ is the volume of $Y$ in string units. The ten-dimensional coupling $\tau_{2} \equiv 1 / g_{(10)}$ and the RR axion $\tau_{1} \equiv c^{0}$ can be combined into the ten-dimensional axio-dilaton field $\tau=\tau_{1}+\mathrm{i} \tau_{2}$.

At string tree level, the metric on $\mathcal{M}_{K}(Y)$ can be obtained from the special Kähler space $\mathcal{K}_{K}(Y)$ via the $c$-map and thus is determined by the prepotential $F\left(X^{\Lambda}\right)$. The latter receives world-sheet instanton corrections which can conveniently be found via the classical mirror map [6, 5]. Its large volume expansion takes the form

$$
F\left(X^{\Lambda}\right)=-\kappa_{a b c} \frac{X^{a} X^{b} X^{c}}{6 X^{0}}+\chi_{Y} \frac{\zeta(3)\left(X^{0}\right)^{2}}{2(2 \pi \mathrm{i})^{3}}-\frac{\left(X^{0}\right)^{2}}{(2 \pi \mathrm{i})^{3}} \sum_{\hat{\gamma}_{+}} n_{q_{a}}^{(0)} \operatorname{Li}_{3}\left(\mathrm{e}^{2 \pi \mathrm{i} q_{a} X^{a} / X^{0}}\right)
$$


where $\hat{\gamma}$ denotes the set of charges $\left\{q_{a}\right\}$ and its subset $\hat{\gamma}_{+}$corresponds to effective homology classes $q_{a} \gamma^{a} \in H_{2}^{+}(Y)$ (i.e., $q_{a} \geq 0$ for all $a$, not all of them vanishing simultaneously). Furthermore, $n_{q_{a}}^{(0)}$ is the genus zero BPS invariant in the homology class $q_{a} \gamma^{a}, \operatorname{Li}_{s}(x)=\sum_{m=1}^{\infty} m^{-s} x^{m}$ is the polylogarithm function, and $\chi_{Y}$ is the Euler number of $Y$. Note that the last two terms in (3.8) may be combined by including the zero class $q_{a}=0$ into the sum and setting $n_{0}^{(0)}=-\chi_{Y} / 2$. Similarly to the Type IIA case, the $c$-map metric on $\mathcal{M}_{K}(Y)$ receives a one-loop correction proportional to the Euler class $\chi_{Y}$.

Classically, i.e., at string tree-level and leading order in the $\alpha^{\prime}$ expansion, $\mathcal{M}_{K}(Y)$ admits an isometry group $\operatorname{SL}(2, \mathbb{R})$, acting on the physical fields as [33, 10]

$$
\begin{gathered}
\tau \mapsto \frac{a \tau+b}{c \tau+d}, \quad t^{a} \mapsto t^{a}|c \tau+d|, \quad c_{a} \mapsto c_{a}, \\
\left(\begin{array}{l}
c^{a} \\
b^{a}
\end{array}\right) \mapsto\left(\begin{array}{ll}
a & b \\
c & d
\end{array}\right)\left(\begin{array}{l}
c^{a} \\
b^{a}
\end{array}\right), \quad\left(\begin{array}{l}
c_{0} \\
\psi
\end{array}\right) \mapsto\left(\begin{array}{cc}
d & -c \\
-b & a
\end{array}\right)\left(\begin{array}{l}
c_{0} \\
\psi
\end{array}\right),
\end{gathered}
$$

with $a d-b c=1$, which is inherited from the $\operatorname{SL}(2, \mathbb{R})$ invariance of the ten-dimensional Type IIB supergravity action. The $\alpha^{\prime}$ - and $g_{s}$-corrections break this symmetry to the discrete group $\mathrm{SL}(2, \mathbb{Z})$, which is expected to be a symmetry of the full quantum theory.

The twistor space $\mathcal{Z}$ of $\mathcal{M}_{K}(Y)$ has the same patch structure as its Type IIA counterpart, cf. Fig. 1. Provided that $Y=\tilde{X}$ is the mirror CY of $X$, it also employs the same transition functions (3.1). Thus, also the twistor lines underlying the perturbative $\mathcal{M}_{K}(\tilde{X})$ and $\mathcal{M}_{C}(X)$ are identical. Using the large volume expansion (3.8) and identifying $\mathcal{R}=\tau_{2} / 2$ (as will become clear in (3.15) below), the contact potential (3.7) has the large volume expansion

$$
\mathrm{e}^{\Phi_{\mathrm{pert}}}=\frac{\tau_{2}^{2}}{2} V\left(t^{a}\right)-\frac{\chi_{Y} \zeta(3)}{8(2 \pi)^{3}} \tau_{2}^{2}+\mathrm{e}^{\Phi_{\mathrm{ws}}}-\frac{\chi_{Y}}{192 \pi}
$$

where

$$
\mathrm{e}^{\Phi_{\mathrm{ws}}}=\frac{\tau_{2}^{2}}{4(2 \pi)^{3}} \sum_{\hat{\gamma}_{+}} n_{q_{a}}^{(0)} \operatorname{Re}\left[\operatorname{Li}_{3}\left(\mathrm{e}^{2 \pi \mathrm{i} q_{a} z^{a}}\right)+2 \pi q_{a} t^{a} \operatorname{Li}_{2}\left(\mathrm{e}^{2 \pi \mathrm{i} q_{a} z^{a}}\right)\right]
$$

is the world-sheet instanton contribution. In the large volume limit, $\mathrm{e}^{\Phi_{\text {pert }}}$ coincides with the four-dimensional dilaton $\phi$, and may in fact be adopted as its definition in the quantum regime.

The classical $\operatorname{SL}(2, \mathbb{R})$-invariance 3.9 ) can be lifted to the twistor space. In this context, it is useful to adapt the twistor line $\alpha^{[i]}$ to the $\operatorname{SL}(2, \mathbb{R})$ symmetry and work with

$$
\alpha_{\mathrm{B}}=-\frac{\mathrm{i}}{4} \alpha_{\mathrm{A}}+\frac{1}{2} \tilde{\xi}_{\Lambda} \xi^{\Lambda}=\alpha+\tilde{\xi}_{\Lambda} \xi^{\Lambda}
$$


Then the $\operatorname{SL}(2, \mathbb{R})$ action on the complex coordinates on the patch $\hat{\mathcal{U}}_{0}$ takes the form 19.

$$
\begin{aligned}
\xi^{0} & \mapsto \frac{a \xi^{0}+b}{c \xi^{0}+d}, \quad \xi^{a} \mapsto \frac{\xi^{a}}{c \xi^{0}+d}, \quad \tilde{\xi}_{a} \mapsto \tilde{\xi}_{a}+\frac{\mathrm{i} c}{4\left(c \xi^{0}+d\right)} \kappa_{a b c} \xi^{b} \xi^{c} \\
\tilde{\xi}_{0} & \mapsto\left(c \xi^{0}+d\right) \tilde{\xi}_{0}-c \alpha_{\mathrm{B}}+c \xi^{a} \tilde{\xi}_{a}+\frac{\mathrm{i} c^{2}}{12} \frac{\kappa_{a b c} \xi^{a} \xi^{b} \xi^{c}}{c \xi^{0}+d} \\
\alpha_{\mathrm{B}} & \mapsto \frac{\alpha_{\mathrm{B}}}{c \xi^{0}+d}+\frac{\mathrm{i} c}{12} \frac{\kappa_{a b c} \xi^{a} \xi^{b} \xi^{c}}{\left(c \xi^{0}+d\right)^{2}} .
\end{aligned}
$$

Under the action (3.13), the complex contact one-form transforms by an overall holomorphic factor $\mathcal{X}^{[i]} \rightarrow \mathcal{X}^{[i]} /\left(c \xi^{0}+d\right)$ so that the complex contact structure remains invariant.

The holomorphic contact action (3.13) on $\mathcal{Z}$ decomposes into the isometric action (3.9) on $\mathcal{M}_{K}(Y)$ and a $\mathrm{SU}(2)$ rotation on the fiber. The latter is given by the following transformation of the fiber coordinate $\mathbf{z}$ :

$$
\mathbf{z} \mapsto \frac{c \tau_{2}+\mathbf{z}\left(c \tau_{1}+d\right)+\mathbf{z}|c \tau+d|}{\left(c \tau_{1}+d\right)+|c \tau+d|-\mathbf{z} c \tau_{2}}
$$

The $\mathrm{SL}(2, \mathbb{R})$ transformation of the complex coordinates $\xi^{\Lambda}, \tilde{\xi}_{\Lambda}^{[0]}, \alpha_{\mathrm{B}}^{[0]}$, given by eq. (3.13), should be consistent with the transformation of the (classical part of the) twistor lines (3.4) induced by the transformation laws (3.9) and (3.14). This condition allows to determine the classical relation between the physical Type IIA fields $\left(\mathcal{R}, z^{a}, \zeta^{\Lambda}, \tilde{\zeta}_{\Lambda}, \sigma\right)$ and their Type IIB counterparts $\left(\tau, b^{a}, t^{a}, c^{a}, c_{a}, c_{0}, \psi\right)$ 19

$$
\begin{aligned}
& \mathcal{R}=\frac{1}{2} \tau_{2}, \quad Y^{a}=\frac{1}{2} \tau_{2} z^{a}, \quad \zeta^{0}=\tau_{1}, \quad \zeta^{a}=-\left(c^{a}-\tau_{1} b^{a}\right), \\
& \tilde{\zeta}_{a}=c_{a}+\frac{1}{2} \kappa_{a b c} b^{b}\left(c^{c}-\tau_{1} b^{c}\right), \quad \tilde{\zeta}_{0}=c_{0}-\frac{1}{6} \kappa_{a b c} b^{a} b^{b}\left(c^{c}-\tau_{1} b^{c}\right), \\
& \sigma=-2\left(\psi+\frac{1}{2} \tau_{1} c_{0}\right)+c_{a}\left(c^{a}-\tau_{1} b^{a}\right)-\frac{1}{6} \kappa_{a b c} b^{a} c^{b}\left(c^{c}-\tau_{1} b^{c}\right) .
\end{aligned}
$$

This relation constitutes the classical limit of the generalized mirror map and agrees with the identification found via the dimensional reduction of the classical ten-dimensional Type IIB supergravity action on $Y$ [33, 10]. The explicit construction of the quantum version of this map, including the perturbative as well as the worldsheet, $\mathrm{D}(-1)$ and D1-instanton corrections, will be the subject of Section 5 .

\section{Instanton corrected Type IIB HM moduli space}

We now proceed by dressing up the perturbative Type IIB twistor space description of the last subsection by including $\mathrm{D}(-1)$ and D1-instanton corrections. In this case 
$\mathcal{M}_{K}(Y)$ still possesses $d+1$ commuting isometries ${ }^{5}$ and thus falls into the class of toric QK spaces discussed in Section 2. The corresponding instanton corrections were found by carrying out an $\mathrm{SL}(2, \mathbb{Z})$ completion of the so-called "tensor potential" [35, which, up to the scale factor, corresponds to the contact potential $(2.10)$ in the twistor construction. Here we provide the complete description of the instanton corrected twistor space including the transition functions and the twistor lines. The gauge equivalence between this novel formulation and the Type IIA twistor space description 19 is established in Appendix $\mathrm{A}$ with more computational details relegated to Appendix B.

Adapting the results [16], the instanton corrected Type IIB contact potential is expressed in terms of a generalized Eisenstein series

$$
\mathrm{e}^{\Phi_{\text {IIB }}}=\frac{\tau_{2}^{2}}{2} V\left(t^{a}\right)+\frac{\sqrt{\tau_{2}}}{8(2 \pi)^{3}} \sum_{q_{a} \geq 0} n_{q_{a}}^{(0)} \sum_{m, n}^{\prime} \frac{\tau_{2}^{3 / 2}}{|m \tau+n|^{3}}\left(1+2 \pi|m \tau+n| q_{a} t^{a}\right) \mathrm{e}^{-S_{m, n, q_{a}}} .
$$

Here the prime indicates that the $(m, n)$-sum excludes $(0,0)$. Furthermore,

$$
S_{m, n, q_{a}}=2 \pi q_{a}\left(|m \tau+n| t^{a}-\mathrm{i} m c^{a}-\mathrm{i} n b^{a}\right)
$$

is the classical action of a $(p, q)$-string (or rather $(m, n)$-string) wrapped on the 2cycle $q_{a} \gamma^{a} \in H_{2}(Y, \mathbb{Z})$ and the $n_{q_{a}}^{(0)}$ are the BPS invariants introduced in (3.8). The contribution of the $\mathrm{D}(-1)$-instantons is recovered as the $\left(q_{a}=0\right)$-sector of the sum. Observe that the contact potential is actually independent of the RR fields $c_{a}, c_{0}$ and the NS axion $\psi$. With respect to the $\mathrm{SL}(2, \mathbb{Z})$-transformations (3.9) it transforms as a modular form $\mathrm{e}^{\Phi_{\text {IIB }}} \mapsto \mathrm{e}^{\Phi_{\text {IIB }}} /|c \tau+d|$.

The projective superspace description [12] suggests that the instanton terms in (4.1) can be captured by the holomorphic function ${ }^{6}$

$$
G_{\mathrm{IIB}}(\xi)=-\frac{\mathrm{i}}{(2 \pi)^{3}} \sum_{q_{a} \geq 0} n_{q_{a}}^{(0)} \sum_{\substack{n \in \mathbb{Z} \\ m>0}} \frac{e^{-2 \pi \mathrm{i} m q_{a} \xi^{a}}}{m^{2}\left(m \xi^{0}+n\right)},
$$

with $\xi^{\Lambda}(\mathbf{z})$ given by (3.4). This function has a dense set of poles on the real axis

$$
\mathbf{z}_{ \pm}^{m, n}=\frac{m \tau_{1}+n \mp|m \tau+n|}{m \tau_{2}}, \quad \mathbf{z}_{+}^{m, n} \mathbf{z}_{-}^{m, n}=-1
$$

corresponding to the zeros of $m \xi^{0}+n$ expressed in terms of Type IIB fields using (3.15) (see (4.6) below). For $m>0$, the poles satisfy $\mathbf{z}_{+}^{m, n}<0$ and $\mathbf{z}_{-}^{m, n}>0$, respectively.

\footnotetext{
${ }^{5}$ These are broken once D3, D5 and NS5-brane corrections are included.

${ }^{6}$ Here and in the following, for $q_{a}=0$ the sum over $n$ is defined by first summing the contributions of $n$ and $-n$ which leads to the asymptotics $\sim n^{-2}$.
} 

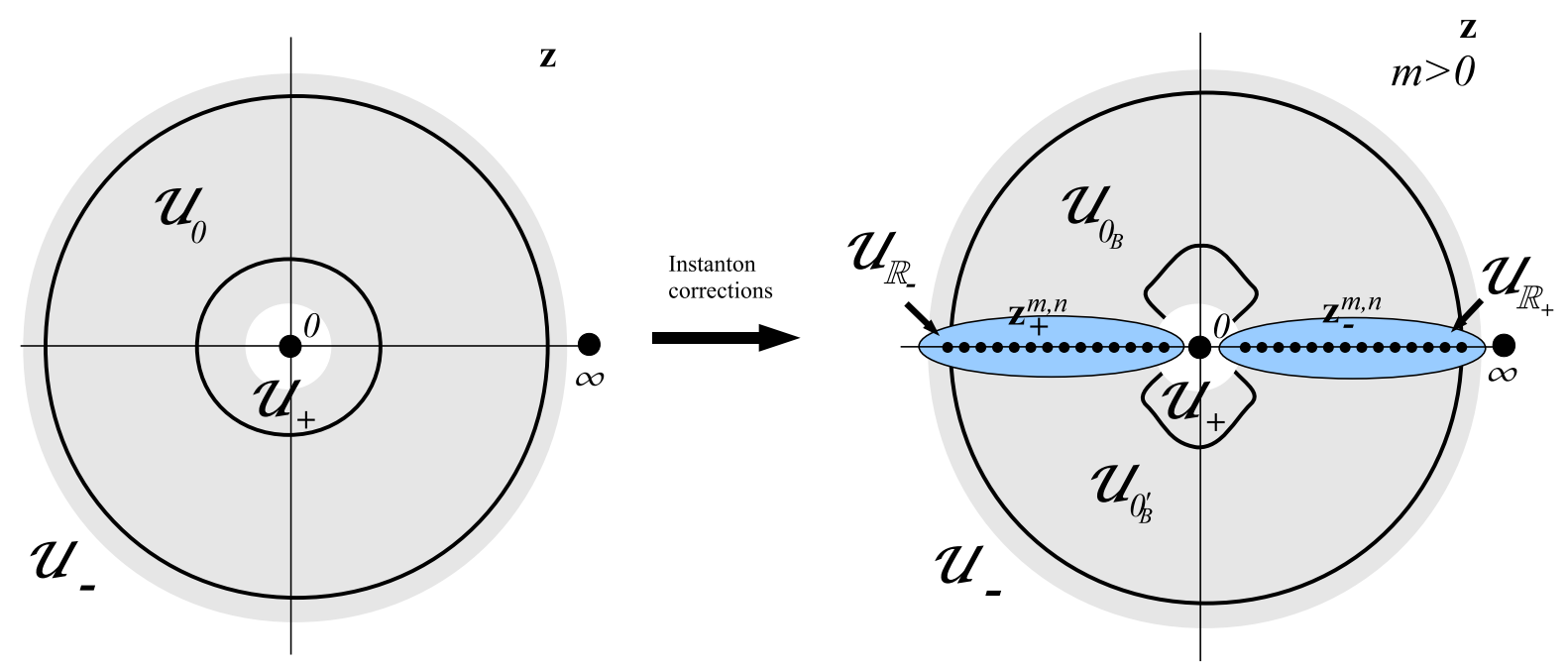

Figure 1: Transition from the perturbative to the instanton corrected Type IIB twistor space. The corrections are encoded in the poles $\mathbf{z}_{ \pm}^{m, n}$ located in the new patches $\mathcal{U}_{\mathbb{R}_{ \pm}}$. This should be compared to the gauge-equivalent Type IIA twistor space shown in Fig. 2, which encodes the instanton corrections in two BPS rays along the imaginary axis.

Each pole captures the contribution of a particular $(m, n)$-instanton configuration. This can easily be seen by noting that the residue of $G_{\mathrm{IIB}}$ at $\mathbf{z}_{ \pm}^{m, n}$ gives rise to exponential terms containing the corresponding instanton action. The "off-shell" $G_{\text {IIB }}$ does not transform as a modular form under $\mathrm{SL}(2, \mathbb{Z})$, however.

The inclusion of the instanton corrections in the twistor space description is then depicted in Fig. 1. The $\mathbb{C} P^{1}$ is covered by six patches: the two (classical) patches $\mathcal{U}_{ \pm}$ surround the north and south poles, two patches $\mathcal{U}_{\mathbb{R}_{\mp}}$ encircle the negative and positive real axis and contain all poles $\mathbf{z}_{ \pm}^{m, n}$, and the remaining two patches $\mathcal{U}_{0_{B}}, \mathcal{U}_{0_{B}^{\prime}}$ cover the upper and lower half-planes of the $\mathbf{z}$-plane, respectively. ${ }^{7}$ The transition functions on the overlaps including $\mathcal{U}_{0_{B}}$ are

$$
\begin{array}{rlrl}
H^{\left[0_{B}+\right]} & =-\frac{\mathrm{i}}{2} F\left(\xi^{\Lambda}\right), & H^{\left[0_{B}-\right]} & =-\frac{\mathrm{i}}{2} \bar{F}\left(\xi^{\Lambda}\right), \\
H^{\left[0_{B} \mathbb{R}_{-}\right]}=-\frac{\mathrm{i}}{2} G_{\mathrm{IIB}}\left(\xi^{\Lambda}\right), & H^{\left[0_{B} \mathbb{R}_{+}\right]} & =-\frac{\mathrm{i}}{2} \bar{G}_{\mathrm{IIB}}\left(\xi^{\Lambda}\right),
\end{array}
$$

The ones on the overlap with $\mathcal{U}_{0_{B}^{\prime}}$ are given by the same expressions and follow from replacing $0_{B} \mapsto 0_{B}^{\prime}$ in (4.5). The anomalous dimensions $c_{\Lambda}, c_{\alpha}$ vanish identically since

\footnotetext{
${ }^{7}$ Strictly speaking, this description is not satisfactory because the poles are dense on the real axis and, in particular, accumulate near $\mathbf{z}=0$. To make the construction rigorous, one should cut off the sum over $m, n$ in (4.3) to $m \leq M,|n| \leq N$. This defines a regularized twistor space, which converges to the desired space in the limit $M, N \rightarrow \infty$.
} 
the perturbative one-loop contribution $c_{\alpha}$, eq. (3.1), is now incorporated as a part of the $\left(q_{a}=0\right)$-sector of the sum (4.3). (An explanation of how this works can be found in Appendix A.2.) This data completely specifies the twistor space underlying the Type IIB HM moduli space.

Our next task is to compute the Type IIB twistor lines, including the instanton corrections, by expressing $\xi^{\Lambda}, \tilde{\xi}_{\Lambda}, \alpha_{\mathrm{B}}$ in terms of the physical Type IIB fields and the fiber coordinate $\mathbf{z}$. The twistor line $\xi^{\Lambda}$ can be obtained from (2.5) by first going to the Type IIA variables via (3.6) and subsequently applying the mirror map (3.15)

$$
\xi^{0}=\tau_{1}+\frac{\tau_{2}}{2}\left(\mathbf{z}^{-1}-\mathbf{z}\right), \quad \xi^{a}=-\left(c^{a}-\tau_{1} b^{a}\right)+\frac{\tau_{2}}{2}\left(z^{a} \mathbf{z}^{-1}-\bar{z}^{a} \mathbf{z}\right) .
$$

The off-shell formulation [18, 12] thereby guarantees, that the relation between the physical fields entering into $\xi^{\Lambda}$ does not receive quantum corrections from $\mathrm{D}(-1)$ and D1-instantons. As a crosscheck, one can also compute the contact potential (2.10) from the transition functions (4.5) with $\xi^{\Lambda}$ given above and verify that this indeed reproduces (4.1). This provides an independent derivation of this result using twistor techniques.

The construction of the twistor lines $\tilde{\xi}_{\Lambda}$ and $\alpha_{\mathrm{B}}$ proceeds by substituting the transition functions (4.5) (depending on (4.6)) into (2.9). A straightforward, although tedious, computation yields the twistor lines in the patch $\mathcal{U}_{0_{B}}$

$$
\begin{aligned}
\tilde{\xi}_{a}^{\left[0_{B}\right]}= & \frac{\mathrm{i}}{2}\left(\tilde{\zeta}_{a}+\frac{\tau_{2}}{2}\left(\mathbf{z}^{-1} F_{a}(z)-\mathbf{z} \bar{F}_{a}(\bar{z})\right)\right)+\frac{\mathrm{i}}{16 \pi^{2}} \sum_{q_{a} \geq 0} n_{q_{a}}^{(0)} q_{a} \sum_{\substack{n \in \mathbb{Z} \\
m \neq 0}} \frac{\mathrm{e}^{-S_{m, n, q_{a}}}}{m|m \tau+n|} \frac{\mathbf{z}_{+}^{m, n}+\mathbf{z}}{\mathbf{z}_{+}^{m, n}-\mathbf{z}}, \\
\tilde{\xi}_{0}^{\left[0_{B}\right]}= & \frac{\mathrm{i}}{2}\left(\tilde{\zeta}_{0}+\frac{\tau_{2}}{2}\left(\mathbf{z}^{-1} F_{0}(z)-\mathbf{z} \bar{F}_{0}(\bar{z})\right)\right)+\frac{1}{32 \pi^{3}} \sum_{q_{a} \geq 0} n_{q_{a}}^{(0)} \sum_{\substack{n \in \mathbb{Z} \\
m \neq 0}} \frac{\mathrm{e}^{-S_{m, n, q_{a}}}}{m|m \tau+n|^{2}} \\
& \times\left\{-\frac{2 \mathbf{z}_{+}^{m, n} \mathbf{z}}{\left(\mathbf{z}-\mathbf{z}_{+}^{m, n}\right)^{2}}+\frac{\mathbf{z}_{+}^{m, n}+\mathbf{z}}{\mathbf{z}_{+}^{m, n}-\mathbf{z}}\left[\frac{m \tau_{1}+n}{|m \tau+n|}+\mathrm{i} \pi m \tau_{2} q_{a}\left(\frac{z^{a}}{\mathbf{z}_{+}^{m, n}}+\bar{z}^{a} \mathbf{z}_{+}^{m, n}\right)\right]\right\} \\
\alpha_{\mathrm{B}}^{\left[0_{B}\right]}= & \frac{1}{4 \mathrm{i}}\left[\sigma+\frac{\tau_{2}}{2}\left(\mathbf{z}^{-1} W(z)-\mathbf{z} \bar{W}(\bar{z})\right)\right. \\
& \left.-\left(\zeta^{\Lambda}+\frac{\tau_{2}}{2}\left(\mathbf{z}^{-1} z^{\Lambda}-\mathbf{z} \bar{z}^{\Lambda}\right)\right)\left(\tilde{\zeta}_{\Lambda}+\frac{\tau_{2}}{2}\left(\mathbf{z}^{-1} F_{\Lambda}(z)-\mathbf{z} \bar{F}_{\Lambda}(\bar{z})\right)\right)\right] \\
& -\frac{1}{32 \pi^{3}} \sum_{q_{a} \geq 0} n_{q_{a}}^{(0)} \sum_{\substack{n \in \mathbb{Z} \\
m \neq 0}} \frac{\mathrm{e}^{-S_{m, n, q a}}}{m^{2}|m \tau+n|}\left\{\frac{\mathbf{z}_{+}^{m, n}+\mathbf{z}}{\mathbf{z}_{+}^{m, n}-\mathbf{z}}\right. \\
& \left.-\frac{m \tau_{2}\left(m \tau_{1}+n\right)}{2|m \tau+n|^{2}}\left(\mathbf{z}^{-1}+\mathbf{z}\right)-\pi q_{a} t^{a} \frac{m^{2} \tau_{2}^{2}}{|m \tau+n|}\left(\frac{\mathbf{z}_{+}^{m, n}}{\mathbf{z}}-\frac{\mathbf{z}}{\mathbf{z}_{+}^{m, n}}\right)\right\}
\end{aligned}
$$

The result is expressed in terms of Type IIB fields except for the coordinates $\tilde{\zeta}_{\Lambda}$ and $\sigma$ which coincide with the physical Type IIA fields. This result follows from the gauge 
equivalence of the Type IIB twistor space description utilized here with the Type IIA construction of [19], which is demonstrated in Appendices $\mathrm{A}$ and B. It shows that the two twistor spaces are related by a set of gauge transformations and thus represent two different descriptions of one and the same twistor geometry.

In the presence of instanton corrections, the classical mirror map is insufficient to relate the remaining Type IIA coordinates $\tilde{\zeta}_{\Lambda}$ and $\sigma$ to the Type IIB fields. The correct relation requires a generalization of (3.15) and is provided by the non-perturbative mirror map constructed in the next section. Together with this map, (4.7) then provides the twistor lines underlying the Type IIB hypermultiplet moduli space.

\section{The non-perturbative mirror map}

As was shown in the previous section, the mirror map between the fields entering into the twistor lines $\xi^{\Lambda}$ does not receive quantum corrections from worldsheet, $\mathrm{D}(-1)$ and D1-instantons. On the other hand, the quantum corrections to the twistor lines $\tilde{\xi}^{\Lambda}, \alpha_{\mathrm{B}}$ suggest that the map relating the physical Type IIA fields $\tilde{\zeta}_{\Lambda}, \sigma$ to $c_{\Lambda}, \psi$ on the Type IIB side will be subject to similar corrections. The aim of this section is to find the explicit form of this map. In this course, we follow the same strategy employed in the construction of the classical mirror map (3.15), demanding consistency between the holomorphic action of $\operatorname{SL}(2, \mathbb{Z})$ on the complex coordinates $\xi^{\Lambda}, \tilde{\xi}_{\Lambda}, \alpha_{\mathrm{B}}$ and the transformation of the Type IIB twistor lines inherited from the physical Type IIB fields and the coordinate $\mathbf{z}$. As we will see, this condition determines the non-perturbative mirror map between the physical Type IIA and IIB fields uniquely.

The key ingredient in the construction is the transformation of the Type IIB fields under $\operatorname{SL}(2, \mathbb{Z})$. Classically, the transformation properties are inherited from the dimensional reduction of the ten-dimensional supergravity action, and we impose that the physical fields parameterizing the quantum corrected $\mathcal{M}_{K}(Y)$ transform according to the classical transformation rules (3.9). In a sense, this corresponds to requiring that the $\mathrm{SL}(2, \mathbb{Z})$-transformations are realized "off-shell", by defining the physical fields as the ones which obey these transformation laws also at the quantum level. By demanding that the $\operatorname{SL}(2, \mathbb{Z})$ acts holomorphically on $\xi^{\Lambda}$, one then establishes that the $\operatorname{SL}(2$, $\mathbb{Z}$ )-transformation of $\mathbf{z}$ is also uncorrected and thus given by (3.14).

In the next step, we turn to the twistor lines (4.7) and read $\tilde{\zeta}_{\Lambda}, \sigma$ as a priori undetermined functions of the physical Type IIB fields, which, in the classical limit, reduce to (3.15). Subsequently, we apply the $\mathrm{SL}(2, \mathbb{Z})$ transformations (3.9) and (3.14) and impose that the transformed expressions can again be expressed in terms of the holomorphic twistor lines. This condition suffices to determine the functions $\tilde{\zeta}_{\Lambda}, \sigma$ uniquely. Since their actual derivation is highly technical and not very illuminating, 
we will proceed along another route by first "guessing" the correct answer and then showing that the resulting Type IIB twistor lines indeed transform holomorphically. The generalized mirror map obtained this way is

$$
\begin{aligned}
& \mathcal{R}=\frac{1}{2} \tau_{2}, \quad z_{\mathrm{IIA}}^{a}=z_{\mathrm{IIB}}^{a}, \quad \zeta^{0}=\tau_{1}, \quad \zeta^{a}=-\left(c^{a}-\tau_{1} b^{a}\right) \\
& \tilde{\zeta}_{a}=c_{a}+\frac{1}{2} \kappa_{a b c} b^{b}\left(c^{c}-\tau_{1} b^{c}\right)+\tilde{\zeta}_{a}^{\text {inst }}, \quad \tilde{\zeta}_{0}=c_{0}-\frac{1}{6} \kappa_{a b c} b^{a} b^{b}\left(c^{c}-\tau_{1} b^{c}\right)+\tilde{\zeta}_{0}^{\text {inst }}, \\
& \sigma=-2\left(\psi+\frac{1}{2} \tau_{1} c_{0}\right)+c_{a}\left(c^{a}-\tau_{1} b^{a}\right)-\frac{1}{6} \kappa_{a b c} b^{a} c^{b}\left(c^{c}-\tau_{1} b^{c}\right)+\sigma^{\text {inst }}
\end{aligned}
$$

with the novel instanton correction terms given by

$$
\begin{aligned}
\tilde{\zeta}_{a}^{\text {inst }}= & \frac{1}{8 \pi^{2}} \sum_{q_{a} \geq 0} n_{q_{a}}^{(0)} q_{a} \sum_{\substack{n \in \mathbb{Z} \\
m \neq 0}} \frac{m \tau_{1}+n}{m|m \tau+n|^{2}} \mathrm{e}^{-S_{m, n, q_{a}}} \\
\tilde{\zeta}_{0}^{\text {inst }}= & -\frac{\mathrm{i}}{16 \pi^{3}} \sum_{q_{a} \geq 0} n_{q_{a}}^{(0)} \sum_{\substack{n \in \mathbb{Z} \\
m \neq 0}}\left[\frac{\left(m \tau_{1}+n\right)^{2}}{|m \tau+n|^{3}}+2 \pi q_{a}\left(t^{a}-\mathrm{i} b^{a} \frac{m \tau_{1}+n}{|m \tau+n|}\right)\right] \frac{\mathrm{e}^{-S_{m, n, q_{a}}}}{m|m \tau+n|} \\
\sigma^{\text {inst }}= & \tau_{1} \tilde{\zeta}_{0}^{\text {inst }}-\left(c^{a}-\tau_{1} b^{a}\right) \tilde{\zeta}_{a}^{\text {inst }}+\frac{\mathrm{i} \tau_{2}^{2}}{8 \pi^{2}} \sum_{q_{a} \geq 0} n_{q_{a}}^{(0)} q_{a} t^{a} \sum_{n \neq 0} \frac{\mathrm{e}^{-S_{0, n, q_{a}}}}{n|n|} \\
& -\frac{\mathrm{i}}{8 \pi^{3}} \sum_{q_{a} \geq 0} n_{q_{a}}^{(0)} \sum_{\substack{n \in \mathbb{Z} \\
m \neq 0}}\left(2-\frac{\left(m \tau_{1}+n\right)^{2}}{|m \tau+n|^{2}}\right) \frac{\left(m \tau_{1}+n\right) \mathrm{e}^{-S_{m, n, q_{a}}}}{m^{2}|m \tau+n|^{2}}
\end{aligned}
$$

This map constitutes the main result of our paper. Notice that only the relations between the $\tilde{\zeta}_{\Lambda}, \sigma$ and $c_{\Lambda}, \psi$ are subject to corrections. In particular the classical mirror map $z_{\mathrm{IIA}}^{a}=z_{\mathrm{IIB}}^{a}$, which relates the complex structure moduli to the complexified Kähler moduli, remains uncorrected. Furthermore, since expressing the correction terms in terms of the Type IIA fields involves the uncorrected relations in (5.1) only, the map is easily inverted to give the physical Type IIB fields in terms of the Type IIA coordinates.

In order to show that this is indeed the correct relation, we first substitute (5.1) into (4.7). This gives the final expression for the Type IIB twistor lines:

$$
\begin{aligned}
\tilde{\xi}_{a}^{\left[0_{B}\right]}= & \frac{\mathrm{i}}{2} c_{a}+\frac{\mathrm{i}}{4} \kappa_{a b c} b^{b}\left(c^{c}-\tau_{1} b^{c}\right)-\frac{\mathrm{i} \tau_{2}}{8} \kappa_{a b c}\left(\mathbf{z}^{-1} z^{b} z^{c}-\mathbf{z} \bar{z}^{b} \bar{z}^{c}\right) \\
& +\frac{\mathrm{i} \tau_{2}}{16 \pi^{2}} \sum_{q_{a} \geq 0} n_{q_{a}}^{(0)} q_{a} \sum_{m, n}^{\prime} \frac{1+\mathbf{z}_{+}^{m, n} \mathbf{z}}{\mathbf{z}-\mathbf{z}_{+}^{m, n}} \frac{\mathrm{e}^{-S_{m, n, q_{a}}}}{|m \tau+n|^{2}}
\end{aligned}
$$




$$
\begin{aligned}
\tilde{\xi}_{0}^{\left[0_{B}\right]}= & \frac{\mathrm{i}}{2} c_{0}-\frac{\mathrm{i}}{12} \kappa_{a b c} b^{a} b^{b}\left(c^{c}-\tau_{1} b^{c}\right)+\frac{\mathrm{i} \tau_{2}}{24} \kappa_{a b c}\left(\mathbf{z}^{-1} z^{a} z^{b} z^{c}-\mathbf{z} \bar{z}^{a} \bar{z}^{b} \bar{z}^{c}\right) \\
& +\frac{\tau_{2}}{32 \pi^{3}} \sum_{q_{a} \geq 0} n_{q_{a}}^{(0)} \sum_{m, n}^{\prime}\left(\frac{1}{m \xi^{0}+n}+\frac{m \tau_{1}+n}{|m \tau+n|^{2}}\right) \frac{1+\mathbf{z}_{+}^{m, n} \mathbf{z}}{\mathbf{z}-\mathbf{z}_{+}^{m, n}} \frac{\mathrm{e}^{-S_{m, n, q_{a}}}}{|m \tau+n|^{2}} \\
& +\frac{\tau_{2}}{16 \pi^{2}} \sum_{q_{a} \geq 0} n_{q_{a}}^{(0)} q_{a} \sum_{m, n}^{\prime}\left(t^{a} \frac{1-\mathbf{z}_{+}^{m, n} \mathbf{z}}{\mathbf{z}-\mathbf{z}_{+}^{m, n}}-\mathrm{i} b^{a} \frac{1+\mathbf{z}_{+}^{m, n} \mathbf{z}}{\mathbf{z}-\mathbf{z}_{+}^{m, n}}\right) \frac{\mathrm{e}^{-S_{m, n, q_{a}}}}{|m \tau+n|^{2}} . \\
\alpha_{\mathrm{B}}^{\left[0_{B}\right]}= & \frac{\mathrm{i}}{2}\left[\psi+c_{\Lambda} \zeta^{\Lambda}+\frac{\tau_{2}}{2} c_{\Lambda}\left(\mathbf{z}^{-1} z^{\Lambda}-\mathbf{z} \bar{z}^{\Lambda}\right)\right]+\frac{\mathrm{i}}{48} \tau \tau_{2} \kappa_{a b c}\left[\mathbf{z}^{-2} z^{a} z^{b} z^{c}+\mathbf{z}^{2} \bar{z}^{a} \bar{z}^{b} \bar{z}^{c}\right] \\
& +\frac{\mathrm{i}}{24} \kappa_{a b c} b^{a}\left[\tau_{2}^{2}\left(3 t^{b} t^{c}+b^{b} b^{c}\right)-\left[2 \zeta^{b}+2 \tau_{2}\left(\mathbf{z}^{-1}-\mathbf{z}\right) b^{b}+3 \mathbf{i} \tau_{2}\left(\mathbf{z}^{-1}+\mathbf{z}\right) t^{b}\right] \zeta^{c}\right] \\
& +\frac{\tau_{2}^{2}}{64 \pi^{3}} \sum_{q_{a} \geq 0} n_{q_{a}}^{(0)} \sum_{m, n}^{\prime}\left(\left(m \tau_{1}+n\right)\left(\mathbf{z}^{-1}-\mathbf{z}\right)-2 m \tau_{2}\right) \frac{1+\mathbf{z}_{+}^{m, n} \mathbf{z}}{\mathbf{z}-\mathbf{z}_{+}^{m, n} \frac{\mathrm{e}^{-S_{m, n, q a}}}{|m \tau+n|^{4}} .}
\end{aligned}
$$

Here we abbreviated $\zeta^{\Lambda}=\left(\tau_{1},-\left(c^{a}-\tau_{1} b^{a}\right)\right)$ together with $z^{\Lambda}=\left(1, b^{a}+\mathrm{i} t^{a}\right)$ for convenience. The $\operatorname{SL}(2, \mathbb{Z})$-transformations of these twistor lines are readily obtained by applying (3.9) and (3.14) together with the intermediate formulas collected in Appendix Q. It turns out, that they transform according to the classical law (3.13). This is highly non-trivial, since the derivation of the mirror map only imposed that the twistor lines transform holomorphically under $\mathrm{SL}(2, \mathbb{Z})$ without specifying the transformation to be of the form (3.13). This establishes that the holomorphic $\mathrm{SL}(2, \mathbb{Z})$-action on the twistor space is realized "off-shell" in a sense that it is not modified in the presence of worldsheet nor $\mathrm{D}(-1)$ and D1-brane instantons. Furthermore, this result confirms the correctness of the found mirror map. The uniqueness of (5.1) can be established by adding additional functions of the Type IIB fields to (5.2). The consistency of the transformations then imposes that these extra contributions have to vanish identically.

\section{Perturbative singularities and instanton corrections}

One of the salient features of the non-perturbative instanton corrections discussed in this paper is their ability to dynamically cure singularities in the perturbative metric on $\mathcal{M}$. The prime example for such a behavior is the conifold singularity which is smoothed out by D2-brane instantons wrapping the shrinking cycle [1, 18]. Motivated by this observation, we will investigate the interplay between the $\mathrm{D}(-1)$ and D1-instanton corrections and the generic singularities of $\mathcal{M}$ induced by the one-loop correction 25, 26]. In this course, we will assume that we work at a regular point in the Kähler/Complex structure moduli space, excluding singularities arising from shrinking (sub-)cycles. Furthermore, our prime focus will be on the $\mathrm{D}(-1)$-corrections and we will comment about the D1-instanton effects only at the end of this section. 


\subsection{The perturbative one-loop singularity}

The perturbatively corrected hypermultiplet metric has been given in [25, 26] and its description in terms of the twistor space was obtained in 24]. In terms of the physical Type IIA fields the metric reads

$$
\begin{aligned}
\mathrm{d} s^{2}= & \frac{r+2 c}{r^{2}(r+c)} \mathrm{d} r^{2}-\frac{1}{r}\left(N^{\Lambda \Sigma}-\frac{2(r+c)}{r K} z^{\Lambda} \bar{z}^{\Sigma}\right)\left(\mathrm{d} \tilde{\zeta}_{\Lambda}-F_{\Lambda \Theta} \mathrm{d} \zeta^{\Theta}\right)\left(\mathrm{d} \tilde{\zeta}_{\Sigma}-\bar{F}_{\Sigma \Xi} \mathrm{d} \zeta^{\Xi}\right) \\
& +\frac{r+c}{16 r^{2}(r+2 c)}\left(\mathrm{d} \sigma+\tilde{\zeta}_{\Lambda} d \zeta^{\Lambda}-\zeta^{\Lambda} \mathrm{d} \tilde{\zeta}_{\Lambda}+4 c \mathcal{A}_{K}\right)^{2}+\frac{4(r+c)}{r} \mathcal{K}_{a \bar{b}} \mathrm{~d} z^{a} \mathrm{~d} \bar{z}^{\bar{b}}
\end{aligned}
$$

where $r=\mathrm{e}^{\phi}, c=-\frac{\chi X}{192 \pi}, N_{\Lambda \Sigma} \equiv \mathrm{i}\left(F_{\Lambda \Sigma}-\bar{F}_{\Lambda \Sigma}\right), \mathcal{K}=-\log K(z, \bar{z})$ is the Kähler potential of the special Kähler base $\mathcal{K}_{K}(X)$ and $\mathcal{A}_{K} \equiv \mathrm{i}\left(\mathcal{K}_{a} \mathrm{~d} z^{a}-\mathcal{K}_{\bar{a}} \mathrm{~d} \bar{z}^{\bar{a}}\right)$ is its Kähler connection.

With respect to the string coupling $r$, the metric possesses three apparent singularities at $r=0, r=-c$ and $r=-2 c$. The last two arise from the one-loop correction and occur in CY compactifications with positive Euler number $\chi_{X}>0$. Notably, the first two points constitute coordinate singularities only. The singularity at $r=0$ can be removed by a simple rescaling of $\zeta^{\Lambda}, \tilde{\zeta}_{\Lambda}$ and $\sigma$ by a power of $r$ and returning to the variable $\phi$, whereas the singularity at $r=-c$ disappears after one trades $r$ for the ten-dimensional string coupling (cf. (3.7))

$$
\tau_{2}=4 e^{\frac{1}{2} \mathcal{K}(z, \bar{z})} \sqrt{r+c} .
$$

This picture is confirmed by computing the quadratic curvature invariant $R_{\mu \nu \rho \sigma} R^{\mu \nu \rho \sigma}$, which diverges at $r=-2 c$ only, and remains regular at $r=-c$ and $r=0$. Thus the only curvature singularity of the perturbatively corrected hypermultiplet metric appears for $\chi_{X}>0$ at $r=-2 c .^{8}$

At the level of the twistor space construction this singularity is caused by the degeneration of the basis of holomorphic $(1,0)$-forms (2.12). Evaluating these for the perturbative twistor lines (3.4) and taking suitable linear combinations, an explicit basis of $(1,0)$-forms is given by [24]

$$
\begin{gathered}
\Pi^{a}=\mathrm{d} z^{a}, \quad \mathcal{Y}_{\Lambda}=\mathrm{d} \tilde{\zeta}_{\Lambda}-F_{\Lambda \Sigma} \mathrm{d} \zeta^{\Sigma}, \\
\Sigma=\mathrm{de}^{\phi}+2 c \mathrm{~d} \log \tau_{2}+\frac{\mathrm{i}}{4}\left[\mathrm{~d} \sigma+\tilde{\zeta}_{\Lambda} \mathrm{d} \zeta^{\Lambda}-\zeta^{\Lambda} \mathrm{d} \tilde{\zeta}_{\Lambda}\right] .
\end{gathered}
$$

The dilaton-direction in (6.1) is generated by the real part of $\Sigma$. This part degenerates at the point where

$$
\mathrm{de}^{\phi}+2 c \mathrm{~d} \log \tau_{2}=0 \quad \bmod \Pi^{a}, \mathcal{Y}_{\Lambda} .
$$

\footnotetext{
${ }^{8}$ This observation is already suggested by Fig. 1 of [36].
} 
Substituting the relation (6.2), one immediately finds that the only solution of (6.4) is given by $r=-2 c$, which clarifies the origin of the perturbative singularity from the twistorial viewpoint.

\subsection{The effect of D-instanton corrections}

In order to analyze the effects of the D-instanton contributions on the perturbative singularity, we first compute the $\mathrm{D}(-1)$ and D1-instanton corrections to (6.3). Starting from the Type IIA twistor lines (A.7) and using the transition functions (A.5) together with (3.2) allows to determine the non-perturbative corrections to $\alpha_{0}^{[+]}, \tilde{\xi}_{\Lambda, 0}^{[+]}$. Substituting the result into (2.12), the instanton-corrected basis of holomorphic (1,0)-forms is given by

$$
\begin{aligned}
\Pi^{a}= & \mathrm{d} z^{a}, \quad \mathcal{Y}_{\Lambda}=\mathrm{d} \tilde{\zeta}_{\Lambda}-F_{\Lambda \Sigma} \mathrm{d} \zeta^{\Sigma}-\frac{\mathrm{i}}{4 \pi^{2}} \sum_{\gamma} n_{\gamma} q_{\Lambda} \mathrm{d} \mathcal{K}_{\gamma} \\
\Sigma= & \mathrm{de}^{\phi}+2 c \mathrm{~d} \log \tau_{2} \\
& +\frac{\mathrm{i}}{4}\left[\mathrm{~d} \sigma+\tilde{\zeta}_{\Lambda} \mathrm{d} \zeta^{\Lambda}-\zeta^{\Lambda} \mathrm{d} \tilde{\zeta}_{\Lambda}-\frac{\mathrm{i}}{8 \pi^{2}} \sum_{\gamma} n_{\gamma} q_{\Lambda}\left(\tau_{2} z^{\Lambda} \mathrm{d} \mathcal{L}_{\gamma}-\overline{\mathcal{L}_{\gamma}} \mathrm{d}\left(\tau_{2} \bar{z}^{\Lambda}\right)\right)\right] .
\end{aligned}
$$

Here

$$
\left.\mathcal{L}_{\gamma} \equiv \frac{\mathrm{i}}{4} \frac{\mathrm{d}}{\mathrm{d} \mathbf{z}} \mathcal{I}_{\gamma}^{(1)}\right|_{\mathbf{z}=0}
$$

denotes the subleading coefficient in the $\mathbf{z}$-expansion of $\mathcal{I}_{\gamma}^{(1)}(\mathbf{z})$ around $\mathbf{z}=0, \mathcal{K}_{\gamma}$ and $\mathcal{I}_{\gamma}^{(1)}$ are defined in (A.8), and $\mathrm{e}^{\phi}$ is the instanton corrected contact potential (A.1). Owed to the relation $q_{\Lambda} z^{\Lambda} \mathcal{L}_{\gamma}=q_{\Lambda} \bar{z}^{\Lambda} \overline{\mathcal{L}_{\gamma}}$, the term appearing in the square bracket in $\Sigma$ is real. Thus the equation controlling the degeneracy of the basis (6.5) is still of the form (6.4), with the perturbative $\mathrm{e}^{\phi}$ now dressed up with instanton corrections.

In order to understand the fate of the perturbative singularity at $r=-2 c$, we need to understand the behavior of $\mathrm{e}^{\phi}$ at strong coupling $\tau_{2} \rightarrow 0$. At this point it is useful to switch to the mirror symmetric Type IIB description (4.1) where we can use $S$-duality to relate $\mathrm{e}^{\phi}$ at strong and weak string coupling. The $\mathrm{D}(-1)$-instanton corrections (leaving out the D1-instanton contribution for the time being) to the four-dimensional dilaton are readily obtained from the second term in (4.1) by setting $q_{a}=0, n_{0}^{(0)}=-\chi_{Y} / 2$. Together with the perturbative worldsheet and string loop corrections, they combine into a real analytic Eisenstein series

$$
\mathrm{e}^{\phi_{\mathrm{D}(-1)}}=-\frac{\chi_{Y} \tau_{2}^{1 / 2}}{16(2 \pi)^{3}} \mathcal{E}_{3 / 2}(\tau, \bar{\tau}), \quad \mathcal{E}_{3 / 2}(\tau, \bar{\tau}):=\sum_{m, n}^{\prime} \frac{\tau_{2}^{3 / 2}}{|m \tau+n|^{3}}
$$


The strong coupling limit of these terms can then be extracted by using the $\mathrm{SL}(2, \mathbb{Z})$ invariance of $\mathcal{E}_{3 / 2}$ and can conveniently be done by first applying an $S$-duality $\tau \mapsto-\tau^{-1}$ to the weak coupling expansion of $\mathcal{E}_{3 / 2}$ and subsequently taking the limit $\tau_{1} \rightarrow 0$

$$
\mathrm{e}^{\phi_{\mathrm{D}(-1)}}=-\frac{\chi_{Y}}{16(2 \pi)^{3}}\left(2 \zeta(3) \tau_{2}^{-1}+4 \zeta(2) \tau_{2}\right)+O\left(\mathrm{e}^{-2 \pi \tau_{2}^{-1}}\right) .
$$

Including the tree-level term, $\mathrm{e}^{\phi}$ then has the strong coupling expansion

$$
\mathrm{e}^{\phi}=-\frac{\chi_{Y}}{16(2 \pi)^{3}}\left(2 \zeta(3) \tau_{2}^{-1}+4 \zeta(2) \tau_{2}\right)+\frac{1}{2} V(t) \tau_{2}^{2}+\ldots,
$$

where the dots stand for terms which are exponentially suppressed. As expected, the strong coupling asymptotics is dominated by the instanton effects. In this asymptotics the four-dimensional dilaton (related to the four-dimensional string coupling via $\mathrm{e}^{-\phi} \propto$ $\left.g_{(4)}^{2}\right)$ behaves as $\mathrm{e}^{\phi} \sim \tau_{2}^{-1}$ so that $g_{(4)} \sim g_{(10)}^{-1 / 2}$. In other words, the four-dimensional coupling is prohibited from divergence: the region of large $g_{(4)}$ is inaccessible on the studied corners of the moduli space.

In the Type IIB description, the perturbative singularity, $r=-2 c$ with $c=\frac{\chi_{Y}}{196 \pi}$, appears for CYs $Y$ (mirror to $X$ ) with $\chi_{Y}<0$. In order to make a statement about the fate of this singularity in the presence of $\mathrm{D}(-1)$-instantons, the condition (6.4) indicates that it is sufficient to consider the asymptotics of $\frac{\mathrm{de}^{\phi}}{\mathrm{d} \log \tau_{2}}$ in the weak and strong coupling regime. For $\tau_{2}$ large (and in the large volume limit), the contact potential is dominated by the classical term so that

$$
\frac{\mathrm{de}^{\phi}}{\mathrm{d} \log \tau_{2}} \underset{\tau_{2} \rightarrow \infty}{\sim} V(t) \tau_{2}^{2}>0 .
$$

At strong coupling, the expansion (6.9) yields

$$
\frac{\mathrm{de}^{\phi}}{\mathrm{d} \log \tau_{2}} \underset{\tau_{2} \rightarrow 0}{\sim} \frac{\zeta(3) \chi_{Y}}{8(2 \pi)^{3}} \tau_{2}^{-1} .
$$

For $\chi_{Y}<0$ the two asymptotics have opposite signs. Therefore the equation (6.4) necessarily has a solution at finite value of $\tau_{2}$. Thus we conclude that the $\mathrm{D}(-1)$ instantons do not resolve the singularity of perturbative hypermultiplet metric.

Before closing this section, let us briefly comment on the effect of the D1-instantons at strong coupling. Their contributions are given by the terms with non-vanishing charge $q_{a}$ in (4.1). In this case, the application of $S$-duality does not lead to terms which are exponentially suppressed as $\tau_{2} \rightarrow 0$, since the $\mathrm{SL}(2, \mathbb{Z})$-transformations also act on the other fields, in particular $t^{a} \mapsto|c \tau+d| t^{a}$. Therefore, one should work directly 
with the double sums in (4.1). The leading contribution at small $\tau_{2}$ (again assuming $\tau_{1}=0$ ) comes from the terms with $n=0$ and is given by

$$
\frac{\tau_{2}^{-1}}{4(2 \pi)^{3}} \sum_{\hat{\gamma}_{+}} n_{q_{a}}^{(0)} \sum_{m=1}^{\infty} \frac{e^{-2 \pi m \tau_{2} q_{a} t^{a}}}{m^{3}} \sim \frac{\zeta(3)}{4(2 \pi)^{3}} \tau_{2}^{-1} \sum_{\hat{\gamma}_{+}} n_{q_{a}}^{(0)} .
$$

In order for the sum over the charges $q_{a}$ to converge, the limit $\tau_{2} \rightarrow 0$ has to be taken by keeping $q_{a} t^{a} \tau_{2}$ fixed and sufficiently large. In this "decompactification limit" one concludes that the D1-instanton contribution to the contact potential has the same asymptotics as the one due to $\mathrm{D}(-1)$-instantons. Keeping $t^{a}$ finite, however, the sum over BPS invariants $n_{q_{a}}^{(0)}$ diverges, so that it is hard to draw any definite conclusions. A proper treatment of this limit will, most likely, involve a resummation of the instanton series, as, e.g., along the lines suggested in [37]. However, in this work we are not embarking on this point.

\section{Discussion and outlook}

The main result of our paper is the non-perturbative mirror map (5.1) which establishes a relation between Type IIA and Type IIB string theory compactified on a generic pair of mirror Calabi-Yau threefolds, taking into account worldsheet, D $(-1)$, and D1instanton corrections. This map constitutes a non-perturbative generalization of the classical limit obtained in [10. In contrast to the classical case, our result is derived from the twistor space description of the corresponding hypermultiplet moduli spaces, thereby avoiding the explicit construction of the underlying QK metrics. Notably, the quantum corrections to the classical map are uniquely determined by the consistent implementation of symplectic covariance (Type IIA) and $\mathrm{SL}(2, \mathbb{Z})$-transformations (Type IIB) on the twistor space. As a spin-off we found that the $\operatorname{SL}(2, \mathbb{Z})$-transformation of the twistor lines does not receive quantum corrections from these non-perturbative effects. We expect that this result will continue to hold once the additional corrections from D3, D5, and NS5-instantons are included. The "off-shell" realization of the SL(2, $\mathbb{Z}$ ) invariance could then provide a powerful tool in unraveling the physical structures underlying these corrections.

A natural question arising from our result concerns the inclusion of these additional corrections in the generalized mirror map. Using the Type IIA formulation, the twistor lines describing D2-branes wrapped on arbitrary three-dimensional special Lagrangian submanifolds have been calculated explicitly in the linear instanton approximation 19 and to all orders in a somewhat implicit form in [20]. The resummation technique of Appendix B can, in principle, be applied to this case as well, thereby providing an interesting generalization of the results reported here. An important test for the consistency 
of the resulting Type IIB twistor lines is then given by their transformation under SL(2, $\mathbb{Z}$ ). In particular the D3-instantons mirror to the B-type D2-instantons are expected to organize themselves into a modular form. This would allow the generalization of our construction, taking these additional corrections into account as well.

Curiously, a "naive" Poisson resummation of the Type IIA twistor lines in the presence of B-type D2-instantons does not lead to Type IIB twistor lines exhibiting the desired behavior under $\operatorname{SL}(2, \mathbb{Z})$. One possible explanation for this intriguing observation is that the Type IIA instanton numbers $n_{\gamma}$ develop a dependence on the resummed charge once all D2-instantons are included. Thus further progress in this direction should go hand in hand with a better understanding of the instanton numbers appearing on the Type IIA side together with their mirrors.

Our second result concerns the singularity structure of the hypermultiplet moduli space. In this context, we found that the singularities in the hypermultiplet moduli space occurring at the perturbative level are not resolved by the inclusion of $\mathrm{D}(-1)$ brane instantons, even though they are part of the same modular invariant. This is, however, in good agreement with the expectation that the dominating non-perturbative contribution at strong coupling should be given by the NS5-brane instantons which have not been included in our analysis. Some progress towards understanding the role of the NS5-brane instantons has been made in [21, 22], but their contribution remains to be fully understood. We hope to return to this point in the future.

\section{Acknowledgements}

The authors are grateful to Boris Pioline and Stefan Vandoren for very useful discussions. The research of S.A. is supported by CNRS. F.S. acknowledges financial support from the ANR grant BLAN06-3-137168 and thanks the LPTA at Montpellier for hospitality while the work was completed.

\section{A. Type IIA and Type IIB twistor spaces and their relation}

Keeping key features as, e.g., the SL(2, $\mathbb{Z})$-invariance on the Type IIB side or symplectic invariance and wall-crossings in Type IIA manifest, naturally leads to twistor space descriptions of instanton corrected HM moduli spaces, which utilize different sets of patches and transition functions. Indeed, the Type IIB description presented in Section 1 is quite different from its Type IIA cousin constructed in [19] and reviewed in Appendix A.1. However, the mirror symmetry indicates that there should be an intrinsic relation between these constructions. In Appendix A.2, we will then show that 
the two constructions are indeed equivalent and related by a gauge-transformation. Technical details of the calculation are further referred to Appendix $B$.

\section{A.1 Instanton corrected Type IIA HM moduli space}

The HM moduli space of Type IIA strings compactified on a CY $X$ receives instanton corrections from D2-branes wrapping the 3-cycles of $X$. The subclass of these D2-instanton corrections wrapping A-cycles is related to $\mathrm{D}(-1)$ and D1-instantons by mirror symmetry [11]. In particular, this implies that the contact potential underlying the Type IIA picture can be obtained by Poisson resumming (4.1) on $n \in \mathbb{Z}$ and subsequently applying the classical mirror map (3.15), which, for the fields appearing in (4.1), does not receive quantum corrections. As a result, one obtains 12

$$
\begin{aligned}
e^{\Phi_{\text {IIA }}}= & \frac{\tau_{2}^{2}}{16} K(z, \bar{z})+\frac{\chi_{X}}{192 \pi} \\
& +\frac{\tau_{2}}{16 \pi^{2}} \sum_{\gamma} n_{\gamma} \sum_{m>0} \frac{\left|q_{\Lambda} z^{\Lambda}\right|}{m} \cos \left(2 \pi m q_{\Lambda} \zeta^{\Lambda}\right) K_{1}\left(2 \pi m \tau_{2}\left|q_{\Lambda} z^{\Lambda}\right|\right) .
\end{aligned}
$$

Matching (4.1) and (A.1) requires the summation over the charge lattice $\gamma=\left\{q_{0}, q_{a}\right\}$ where $q_{0} \in \mathbb{Z}, q_{a} \gamma^{a} \in H_{2}^{+}(Y) \cup H_{2}^{-}(Y) \cup\{0\}$ excluding the case $\gamma=0$, and implies that the instanton numbers $n_{\gamma}$ are related to the genus zero Gopakumar-Vafa invariants of the mirror $C Y$ by

$$
n_{\gamma}=n_{\left(q_{0}, \pm q_{a}\right)} \equiv n_{q_{a}}^{(0)}(Y) \quad \text { for } \quad\left\{q_{a}\right\} \neq 0, \quad n_{\left(q_{0}, 0\right)}=2 n_{0}^{(0)}=\chi_{X}
$$

The twistor space description for these corrections has recently been developed in [19, 20] and the covering underlying the construction is shown in Fig. 2. It consists of the usual patches around the poles $\mathcal{U}_{ \pm}$and two additional patches, $\mathcal{U}_{0_{A}}$ and $\mathcal{U}_{0_{A}^{\prime}}$, which cover the left and the right half-planes of $\mathbb{C} P^{1}$ considered as a complex z-plane. They are separated by two rays joining $\mathbf{z}=0$ and $\mathbf{z}=\infty$ and going along the semi-infinite imaginary axes $\ell_{ \pm} \equiv \mathrm{i} \mathbb{R}^{ \pm}$.

The discontinuities along $\ell_{ \pm}$imply the existence of two transition functions relating $\mathcal{U}_{0_{A}}$ and $\mathcal{U}_{0_{A}^{\prime}}$ since the analytical continuation from one patch to another can be done either through $\ell_{+}$or $\ell_{-}$. These two functions read, respectively, as

$$
H^{\left[00^{\prime}\right]_{+}}=\frac{\mathrm{i}}{2} G_{\mathrm{IIA}}(\xi), \quad H^{\left[00^{\prime}\right]_{-}}=-\frac{\mathrm{i}}{2} \bar{G}_{\mathrm{IIA}}(\xi)
$$

where the function

$$
G_{\text {IIA }}(\xi)=\frac{1}{(2 \pi)^{2}} \sum_{\gamma_{+}} n_{\gamma} \operatorname{Li}_{2}\left(e^{-2 \pi \mathrm{i} q_{\Lambda} \xi^{\Lambda}}\right)
$$



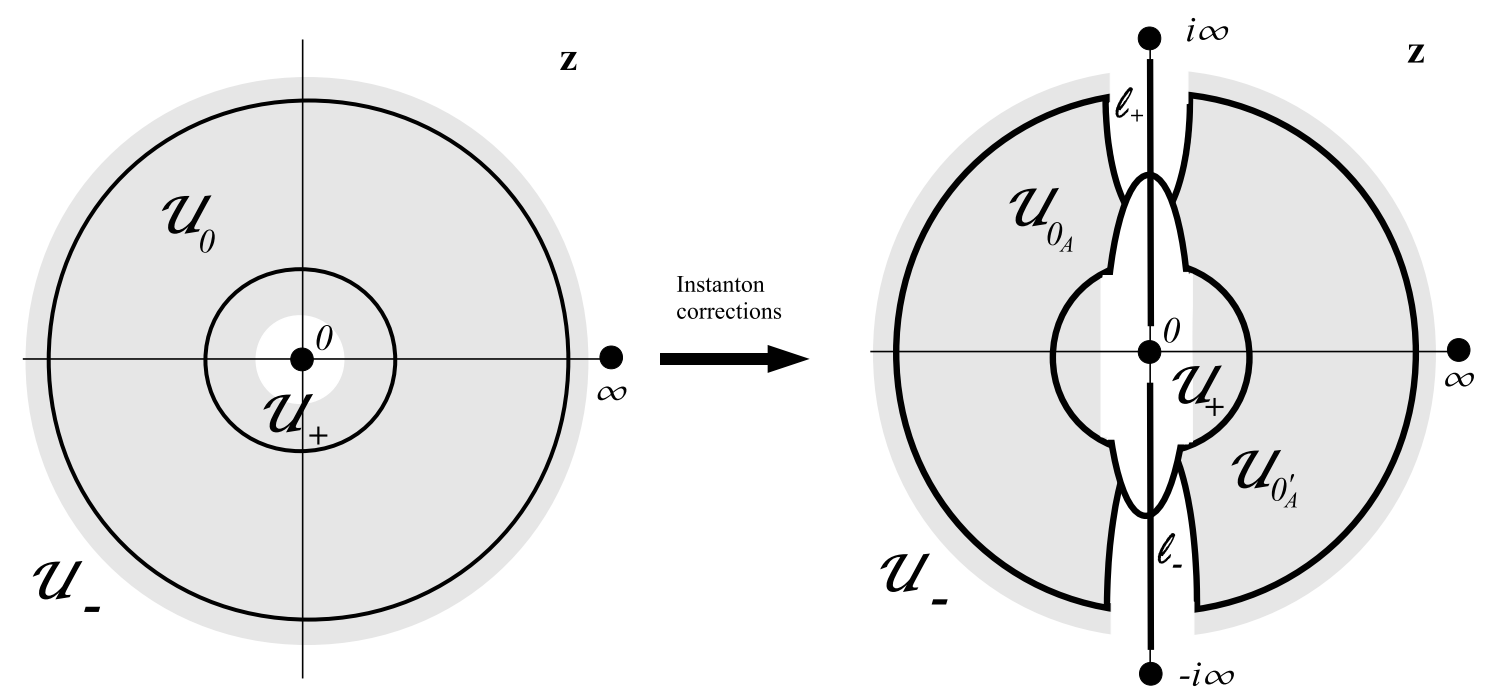

Figure 2: Transition from the classical to the instanton corrected Type IIA twistor space constructed in [19]. The instanton corrections are encoded in the two BPS rays $\ell_{ \pm}$which are covered by an extension of the patches $\mathcal{U}_{ \pm}$. The description is gauge-equivalent to the novel Type IIB twistor space illustrated in Fig. 1.

incorporates the instanton contributions. Here the sum over $\gamma_{+}$is supported on charges $\gamma=\left\{q_{\Lambda}\right\}$ with $\operatorname{Re}\left(q_{\Lambda} z^{\Lambda}\right)>0$. In addition one has to specify the transition functions connecting $\mathcal{U}_{0_{A}}$ (or $\mathcal{U}_{0_{A}^{\prime}}$ ) to $\mathcal{U}_{ \pm}$. They have both perturbative and instanton contributions and read

$$
H^{[+0]}=\frac{\mathrm{i}}{2}(F(\xi)+\mathcal{G}(\xi)), \quad H^{[-0]}=\frac{\mathrm{i}}{2}(\bar{F}(\xi)-\mathcal{G}(\xi)),
$$

where we introduced

$$
\mathcal{G}(\xi)=\frac{\mathrm{i}}{4 \pi^{3}} \sum_{\gamma_{+}} n_{\gamma} \int_{0}^{-\mathrm{i} \infty} \frac{\Xi \mathrm{d} \Xi}{\left(k_{\Lambda} \xi^{\Lambda}\right)^{2}-\Xi^{2}} \mathrm{Li}_{2}\left(e^{-2 \pi \mathrm{i} \Xi}\right) .
$$

Note that the function (A.4) and its conjugate are simply the discontinuities of $\mathcal{G}_{\gamma}$ along the cuts which near the poles can be taken to be along the contours $\ell_{ \pm}$. This property ensures the mutual consistency of the transition functions introduced above and allows to reduce all calculations to the evaluation of integrals along "open contours" $\ell_{ \pm}$[19, 20]. Finally, as in (3.1), there is one non-vanishing anomalous dimension which is $c_{\alpha}=\chi_{X} /(96 \pi)$. It incorporates the effect of the one-loop perturbative contribution found in [25]. Altogether one can check that the presented construction reproduces the contact potential (A.1). 
The twistor lines in the patches $\mathcal{U}_{0_{A}}$ and $\mathcal{U}_{0_{A}^{\prime}}$ have been computed in [19] and are given by

$$
\begin{aligned}
\xi^{\Lambda}= & \zeta^{\Lambda}+\mathcal{R}\left(\mathbf{z}^{-1} z^{\Lambda}-\mathbf{z} \bar{z}^{\Lambda}\right) \\
\tilde{\xi}_{\Lambda}^{\left[0_{A}\right]}= & \frac{\mathrm{i}}{2}\left(\tilde{\zeta}_{\Lambda}+\mathcal{R}\left(\mathbf{z}^{-1} F_{\Lambda}-\mathbf{z} \bar{F}_{\Lambda}\right)\right)+\frac{\mathrm{i}}{32 \pi^{2}} \sum_{\gamma} n_{\gamma} q_{\Lambda} \mathcal{I}_{\gamma}^{(1)}(\mathbf{z}), \\
\alpha_{\mathrm{A}}^{\left[0_{A}\right]}= & \sigma+\mathcal{R}\left(\mathbf{z}^{-1} W-\mathbf{z} \bar{W}\right)+\frac{\mathrm{i} \chi_{X}}{24 \pi} \log \mathbf{z}+\frac{\mathrm{i} \mathcal{R}}{2 \pi^{2}} \sum_{\gamma} n_{\gamma} q_{\Lambda}\left(\mathbf{z}^{-1} z^{\Lambda}+\mathbf{z} \bar{z}^{\Lambda}\right) \mathcal{K}_{\gamma} \\
& +\frac{1}{16 \pi^{2}} \sum_{\gamma} n_{\gamma}\left[\frac{1}{\pi \mathrm{i}} \mathcal{I}_{\gamma}^{(2)}(\mathbf{z})+q_{\Lambda} \xi^{\Lambda} \mathcal{I}_{\gamma}^{(1)}(\mathbf{z})\right],
\end{aligned}
$$

where the sum over $\gamma$ runs over the union of $\gamma_{+}$and $\gamma_{-}$and we introduced

$$
\begin{aligned}
\mathcal{K}_{\gamma} & \equiv \frac{\mathrm{i}}{4} \mathcal{I}_{\gamma}^{(1)}(0)=\sum_{m=1}^{\infty} \frac{1}{m} \sin \left(2 \pi m q_{\Lambda} \zeta^{\Lambda}\right) K_{0}\left(4 \pi m \mathcal{R}\left|q_{\Lambda} z^{\Lambda}\right|\right), \\
\mathcal{I}_{\gamma}^{(\nu)}(\mathbf{z}) & \equiv \sum_{m=1}^{\infty} \sum_{s= \pm 1} \frac{s^{\nu}}{m^{\nu}} e^{-2 \pi \mathrm{i} s m q_{\Lambda} \zeta^{\Lambda}} \int_{0}^{\infty} \frac{\mathrm{d} t}{t} \frac{t-\epsilon_{\gamma} s \mathrm{i} \mathbf{z}}{t+\epsilon_{\gamma} s \mathrm{i} \mathbf{z}} e^{-2 \pi m \epsilon_{\gamma} \mathcal{R} q_{\Lambda}\left(t^{-1} z^{\Lambda}+t \bar{z}^{\Lambda}\right)},
\end{aligned}
$$

with $\epsilon_{\gamma}=\operatorname{sign}\left(\operatorname{Re} q_{\Lambda} z^{\Lambda}\right)$.

We remark that the only effect of the non-vanishing anomalous dimension on the twistor lines is the logarithmic term in $\alpha_{\mathrm{A}}$ which is present in all patches. If the cut of the logarithm is set to be along the imaginary axis, one gets an additional contribution to the transition function $H^{\left[00^{\prime}\right]}$, which is needed to cancel the difference between the two branches of the logarithm on the two sides of the cut. To relate our picture to the Type IIB formulation in the next subsection, it will be convenient to split the logarithmic term into two parts and direct the cut of each term along positive and negative imaginary half-axes, respectively. In this case, the additional contributions $\operatorname{read}^{9}$

$$
H_{\mathrm{an}}^{\left[00^{\prime}\right]_{ \pm}}= \pm \frac{\mathrm{i} \chi_{X}}{96}
$$

This anomalous contribution is important for establishing mirror symmetry at the level of the twistor space.

\section{A.2 Mirror symmetry of the twistor spaces}

We will now show that the two twistor spaces underlying the Type IIA and Type IIB formulations are actually identical, as required by mirror symmetry. More precisely, we

\footnotetext{
${ }^{9} \mathrm{As}$ a consequence of this anomalous contribution, the consistency condition (2.7) picks up an additional constant term when relating patches separated by the logarithmic branch-cut.
} 
demonstrate that they are related by a gauge transformation of the form (2.8). Since the twistor lines $\xi^{\Lambda}$ are already identical in the Type IIA and Type IIB formulation, it suffices to consider gauge transformations of $\tilde{\xi}_{\Lambda}^{[i]}$ and $\alpha_{\mathrm{B}}^{[i]}$

$$
\tilde{\xi}_{\Lambda}^{[i]} \mapsto \tilde{\xi}_{\Lambda}^{[i]}-\partial_{\xi^{\Lambda}} T^{[i]}, \quad \alpha_{\mathrm{B}}^{[i]} \mapsto \alpha_{\mathrm{B}}^{[i]}-T^{[i]} .
$$

Thus, knowing the relation between the twistor lines $\alpha_{\mathrm{B}}$ in the two constructions allows us to directly read off the holomorphic functions underlying the gauge transformation.

In the first step towards establishing this gauge equivalence, we perform a Poisson resummation of the Type IIA twistor lines defined in the patches $\mathcal{U}_{0_{A}}$ and $\mathcal{U}_{0_{A}^{\prime}}$. The details of this resummation can be found in Appendix B. In both patches the result can be written as

$$
\tilde{\xi}_{\Lambda}^{\left[0_{A}\right]}=\tilde{\xi}_{\Lambda}^{\left[0_{B}\right]}+\partial_{\xi^{\Lambda}} \mathcal{T}_{\alpha}, \quad \alpha_{\mathrm{B}}^{\left[0_{A}\right]}=\alpha_{\mathrm{B}}^{\left[0_{B}\right]}+\mathcal{T}_{\alpha}
$$

where $\mathcal{T}_{\alpha}\left(\xi^{\Lambda}\right)$ is given in $(B .25)$. Here the relation is written on the intersection $\mathcal{U}_{0_{A}} \cap \mathcal{U}_{0_{B}}$ which coincides with the second quadrant of the complex z-plane. To write it in other quadrants, it is sufficient to put primes on the patch labels in appropriate places.

This relation between twistor lines has the form of the gauge transformation (A.10) so that it is tempting to immediately apply such interpretation to it. However, this cannot be done straightforwardly because the function $\mathcal{T}_{\alpha}$ is not holomorphic everywhere but has discontinuities along the real and imaginary axes, originating from the sign functions in $(\mathrm{B} .25)$. This is consistent with the fact that $\mathcal{T}_{\alpha}$ arises from the pole at $t= \pm \mathrm{iz}$ in the original integral (A.8), which is responsible for the discontinuity of the Type IIA twistor lines across the rays $\ell_{ \pm}$along the imaginary axis. Similarly, the discontinuity along the real axis accounts for the discontinuity of the Type IIB twistor lines owed to the condensation of poles, as can be seen explicitly from the dual representation of the $\mathcal{T}$-terms in $(\mathrm{B} .20)$.

The presence of these discontinuities suggests that one should refine the covering of the $\mathbb{C} P^{1}$ used in the Type IIA picture by representing $\mathcal{U}_{0_{A}}$ and $\mathcal{U}_{0_{A}^{\prime}}$ as a union of three patches

$$
\mathcal{U}_{0_{A}}=\mathcal{U}_{\mathbb{R}_{-}} \cup \mathcal{U}_{\mathrm{II}} \cup \mathcal{U}_{\mathrm{III}}, \quad \mathcal{U}_{0^{\prime}}=\mathcal{U}_{\mathbb{R}_{+}} \cup \mathcal{U}_{\mathrm{I}} \cup \mathcal{U}_{\mathrm{IV}}
$$

which are related by trivial transition functions. Here $\mathcal{U}_{\mathbb{R}_{ \pm}}$surround the positive and negative real axes and the other patches cover the corresponding quadrants of the $\mathbf{z}^{-}$ complex plane. In each quadrant one can define the holomorphic function $\mathcal{T}_{\alpha}^{[i]}=\mathcal{T}_{\alpha}$, $i \in\{\mathrm{I}, \mathrm{II}, \mathrm{III}, \mathrm{IV}\}$, which then can be analytically continued to the whole plane. It is easy to check that they are given by

$$
\mathcal{T}_{\alpha}^{[\mathrm{I}]}=\frac{\mathrm{i}}{2} G_{\mathrm{A}}^{-}, \quad \mathcal{T}_{\alpha}^{[\mathrm{II}]}=-\frac{\mathrm{i}}{2} G_{\mathrm{A}}^{+}, \quad \mathcal{T}_{\alpha}^{[\mathrm{III}]}=\frac{\mathrm{i}}{2} \bar{G}_{\mathrm{A}}^{-}, \quad \mathcal{T}_{\alpha}^{[\mathrm{IV}]}=-\frac{\mathrm{i}}{2} \bar{G}_{\mathrm{A}}^{+},
$$


where we defined

$$
G_{\mathrm{A}}^{ \pm}\left(\xi^{\Lambda}\right)=\frac{1}{(2 \pi)^{2}} \sum_{ \pm \gamma_{+}} n_{q_{a}}^{(0)} \operatorname{Li}_{2}\left(e^{-2 \pi \mathrm{i} q_{\Lambda} \xi^{\Lambda}}\right)+\frac{\chi_{X}}{96},
$$

with the sum over charges spanning the following lattice

$$
{ }_{ \pm} \gamma_{+}=\left\{\gamma: \operatorname{Re}\left(q_{\Lambda} z^{\Lambda}\right)>0 \text { and } \pm q_{a} t^{a} \geq 0\right\}
$$

This definition implies $G_{\mathrm{A}}^{+}+G_{\mathrm{A}}^{-}=G_{\mathrm{IIA}}+\frac{\chi_{X}}{48}$, so that (A.13) correctly reproduce the discontinuity across the BPS rays $\ell_{ \pm}$.

As a consequence, the relation between twistor lines (A.11) in the four quadrants of the $\mathbf{z}$-plane can be rewritten as

$$
\tilde{\xi}_{\Lambda}^{[i]_{A}}=\tilde{\xi}_{\Lambda}^{[i]_{B}}+\partial_{\xi^{\Lambda}} \mathcal{T}_{\alpha}^{[i]}, \quad \alpha_{\mathrm{B}}^{[i]_{A}}=\alpha_{\mathrm{B}}^{[i]_{B}}+\mathcal{T}_{\alpha}^{[i]}
$$

where the indices $[i]_{A}$ and $[i]_{B}$ denote the restriction of the corresponding Type IIA or Type IIB twistor line to the patch $\mathcal{U}_{i}$. Since every $\mathcal{T}_{\alpha}^{[i]}$ is holomorphic in $\mathcal{U}_{i}$, they now can be interpreted as gauge-transformations $T^{[i]}$ from ( $\mathrm{A.10}$ ) relating $\tilde{\xi}_{\Lambda}^{[i]_{A}}, \alpha_{\mathrm{B}}^{[i]_{A}}$ to $\tilde{\xi}_{\Lambda}^{[i]_{B}}, \alpha_{\mathrm{B}}^{[i]_{B}}$. In this way we recover the Type IIB twistor lines in each of the quadrants.

Such gauge transformations affect essentially all transition functions. Let us first consider the corresponding change of the transition functions between quadrants I and II and between III and IV. To get the complete result, one should also take into account the effect of the anomalous dimension expressed in terms of the additional contribution to $H^{\left[00^{\prime}\right]}(\mathrm{A} .9)$. Then from (A.3), (A.13) and (A.9), the new functions are found to be

$$
\begin{aligned}
H^{[\mathrm{III}]_{B}} & =-\left(H^{\left[00^{\prime}\right]_{+}}+H_{\mathrm{an}}^{\left[00^{\prime}\right]_{+}}\right)+\mathcal{T}_{\alpha}^{[\mathrm{I}]}-\mathcal{T}_{\alpha}^{[\mathrm{II}]}=0, \\
H^{[\mathrm{IIIIV}]_{B}} & =\left(H^{\left[00^{\prime}\right]_{-}}+H_{\mathrm{an}}^{\left[00^{\prime}\right]_{-}}\right)+\mathcal{T}_{\alpha}^{[\mathrm{III}]}-\mathcal{T}_{\alpha}^{[\mathrm{IV}]}=0 .
\end{aligned}
$$

Thus the gauge transformation removes the branch cuts along the BPS rays. Therefore, $\mathcal{U}_{\mathrm{I}}$ and $\mathcal{U}_{\mathrm{II}}$, as well as $\mathcal{U}_{\mathrm{III}}$ and $\mathcal{U}_{\mathrm{IV}}$, can be unified in a single patch, which coincides with $\mathcal{U}_{0_{B}}$ and $\mathcal{U}_{0_{B}^{\prime}}$, respectively.

Next, the transition functions between the quadrants and the patches $\mathcal{U}_{\mathbb{R}_{ \pm}}$become

$$
H^{\left[i \mathbb{R}_{+}\right]_{B}}=\mathcal{T}_{\alpha}^{[i]}, \quad i=\mathrm{I}, \mathrm{IV}, \quad H^{\left[i \mathbb{R}_{-}\right]_{B}}=\mathcal{T}_{\alpha}^{[i]}, \quad i=\mathrm{II}, \mathrm{III}
$$

Comparing the representation $(\overline{B .20})$ of the function $\mathcal{T}_{\alpha}$ with the function $G_{\text {IIB }}$ given in (4.3), one observes that they are almost the same. In this "Type IIB" representation, the difference is due to some sign factors and an additional non-holomorphic term in the exponential of $\mathcal{T}_{\alpha}$. However, performing the Poisson resummation, one finds 
that the difference between these two functions is in fact holomorphic in both patches $\mathcal{U}_{\mathbb{R}_{ \pm}}$. Indeed, the resumed expression for $\mathcal{T}_{\alpha}$ is given in $(B .25)$, whereas the result of resummation of $G_{\text {IIB }}$ immediately follows from the first equation in (B.22) and reads

$$
G_{\mathrm{IIB}}=\frac{\tilde{\varepsilon}}{4 \pi^{2}} \sum_{q_{a} \geq 0} n_{q_{a}}^{(0)}\left(\sum_{\tilde{\varepsilon} q_{0}=1}^{\infty} \operatorname{Li}_{2}\left(\mathrm{e}^{-2 \pi \mathrm{i} q_{\Lambda} \xi^{\Lambda}}\right)+\frac{1}{2} n_{q_{a}}^{(0)} \operatorname{Li}_{2}\left(\mathrm{e}^{-2 \pi \mathrm{i} q_{a} \xi^{a}}\right)\right) .
$$

Then, denoting $x=q_{a} b^{a}$ and $y=q_{a} t^{a}$, for both $i=$ II and III one obtains

$$
\begin{aligned}
\mathcal{T}_{\alpha}^{[i]}+\frac{\mathrm{i}}{2} G_{\text {IIB }}= & \frac{\mathrm{i}}{8 \pi^{2}}\left(\sum_{\hat{\gamma}: x<0, y>0} \sum_{0<q_{0}<x}-\sum_{\hat{\gamma}: x>0, y>0} \sum_{-x<q_{0}<0}\right) n_{q_{a}}^{(0)} \operatorname{Li}_{2}\left(\mathrm{e}^{-2 \pi \mathrm{i} q_{\Lambda} \xi^{\Lambda}}\right) \\
& +\frac{\mathrm{i}}{16 \pi^{2}}\left(\sum_{\hat{\gamma}: x<0, y>0}-\sum_{\hat{\gamma}: x>0, y>0}\right) n_{q_{a}}^{(0)} \operatorname{Li}_{2}\left(e^{-2 \pi \mathrm{i} q_{a} \xi^{a}}\right) .
\end{aligned}
$$

This is a holomorphic function and therefore can be removed by a gauge transformation in the patch $\mathcal{U}_{\mathbb{R}_{-}}$. Similarly, one can show that for $i=\mathrm{I}$ and IV the combination $\mathcal{T}_{\alpha}^{[i]}+\frac{\mathrm{i}}{2} \bar{G}_{\mathrm{IIB}}(\xi)$ is given by the same expression (A.20). Thus, it is also holomorphic and removable by a gauge transformation in the patch $\mathcal{U}_{\mathbb{R}_{+}}$, so that in both cases one recovers the transition functions of the Type IIB formulation.

Finally, it remains to consider the transition functions between the north pole and different quadrants. They are given by

$$
H^{[+i]_{B}}=\frac{\mathrm{i}}{2} F+\frac{\mathrm{i}}{2} \mathcal{G}^{[i]}-\mathcal{T}_{\alpha}^{[i]}
$$

where $\mathcal{G}^{[i]}$ denotes the corresponding holomorphic branch of the function (A.6) in $\mathcal{U}_{i}$. The last two terms coincide with $\frac{i}{2} \mathcal{G}-\mathcal{T}_{\alpha}$. This combination decomposes into two parts. One part is the "constant" term $\frac{i \varepsilon \tilde{\varepsilon}}{192} \chi_{X}$ with discontinuities along real and imaginary axes, while the rest is holomorphic ${ }^{10}$ in $\mathcal{U}_{+} \backslash\left(\mathcal{U}_{\mathbb{R}_{+}} \cup \mathcal{U}_{\mathbb{R}_{-}}\right)$and therefore can be removed by an appropriate gauge transformation. As a result, up to the above "constant" term, the transition functions between the north pole and all quadrants are given by the holomorphic prepotential only. A similar gauge transformation can be performed in the patch $\mathcal{U}_{-}$around the south pole where one finds the same result.

\footnotetext{
${ }^{10}$ In fact, the situation is complicated by the cut along the real axis originating from dependence of $\Delta_{\gamma}$ on $\tilde{\varepsilon}$ in (B.25). However, the entire real axis (except 0 ) is supposed to be covered by $\mathcal{U}_{\mathbb{R}_{+}}$and $\mathcal{U}_{\mathbb{R}_{-}}$ so that formally our function is indeed regular in the domain under consideration. Another way to see this is to use the representation $(\overline{B .20})$ where, instead of the cut, one has infinitely many poles which by assumption belong to $\mathcal{U}_{\mathbb{R}_{+}}$and $\mathcal{U}_{\mathbb{R}_{-}}$. All these complications are in fact a consequence of that the covering used to define the Type IIB twistor space is not regular (see footnote 7 ).
} 
The remaining constant piece then has precisely the correct form to cancel the anomalous dimension which is taken to be vanishing on the Type IIB side. To see this, let us note that the presence of singular terms in the twistor lines due to anomalous dimensions allows for similar singularities in the gauge transformations. In particular, the gauge transformation generated by $T^{[i]}=-\frac{\mathrm{i} \varepsilon \tilde{\varepsilon}}{192} \chi_{X}$ for all patches simply rotates the cuts of the logarithm from the imaginary to the real axis. In our case, such a gauge transformation has been already performed in the four quadrants and, once it is done in $\mathcal{U}_{ \pm}$, it precisely cancels the remaining constant terms in $H^{[ \pm i]_{B}}$. However, this argument requires this gauge transformation to be performed also in $\mathcal{U}_{\mathbb{R}_{ \pm}}$, which would introduce additional terms in $H^{\left[i \mathbb{R}_{ \pm}\right]_{B}}$. Setting these terms to zero "by hand" is equivalent to taking the anomalous dimension to be vanishing. Thus we arrive at the same set of anomalous dimensions and transition functions (4.5) which describes the twistor space of Type IIB HM moduli space.

At the end, we recapitulate the gauge transformations which map the initial Type IIA twistor space into the Type IIB one. They read

$$
T^{[i]}=\mathcal{T}_{\alpha}^{[i]}, \quad T^{[ \pm]}=\mathcal{T}_{\alpha} \mp \frac{\mathrm{i}}{2} \mathcal{G}, \quad T^{\left[\mathbb{R}_{-}\right]}=\mathcal{T}_{\alpha}+\frac{\mathrm{i}}{2} G_{\mathrm{IIB}}, \quad T^{\left[\mathbb{R}_{+}\right]}=\mathcal{T}_{\alpha}+\frac{\mathrm{i}}{2} \bar{G}_{\mathrm{IIB}}
$$

This completes the proof and establishes mirror symmetry between the Type IIA and Type IIB descriptions at the level of the twistor space.

\section{B. Poisson resummation of the twistor lines}

In this appendix we collect the technical details underlying the Poisson resummation of the twistor lines (A.7) with respect to the quantum number $q_{0}$, thereby providing the derivation of eq. (A.11). Inspecting (A.7), we notice that all instanton corrections

are essentially encoded in the functions $\mathcal{I}_{\gamma}^{(\nu)}(\mathbf{z})$. Since the instanton numbers $n_{\gamma}$ are independent of $q_{0}$, one can simplify the calculation by first considering the Poisson resummation of

$$
\begin{aligned}
& \mathcal{I}_{\hat{\gamma}}^{(\nu)}(\mathbf{z}) \equiv \sum_{q_{0} \in \mathbb{Z}} \mathcal{I}_{\gamma}^{(\nu)}(\mathbf{z}) \quad \text { with } \quad \hat{\gamma} \neq 0 \\
& \mathcal{I}_{0}^{(2)}(\mathbf{z}) \equiv \sum_{q_{0} \neq 0} \mathcal{I}_{\gamma}^{(2)}(\mathbf{z}) \quad \text { with } \quad \hat{\gamma}=0,
\end{aligned}
$$

where we have extracted the sum over the charge $q_{0}$ from the lattice sum over $\gamma=$ $\left\{q_{0}, \hat{\gamma}\right\}$. Given these "basic resummations" the other terms entering the twistor lines can be obtained by differentiating with respect to appropriate fields. Thus, we first consider the resummation of $(\overline{B .1})$ before applying the result to the twistor lines in Subsection B.2. 


\section{B.1 Resuming $\mathcal{I}_{\gamma}^{(\nu)}(\mathbf{z})$}

In general Poisson resummation relies on the formula

$$
\sum_{n \in \mathbb{Z}} f(x+n)=\sum_{n \in \mathbb{Z}} \tilde{f}(2 \pi n) e^{2 \pi i n x},
$$

where

$$
f(x)=\frac{1}{2 \pi} \int_{-\infty}^{\infty} d w \tilde{f}(w) e^{i w x}, \quad \tilde{f}(w)=\int_{-\infty}^{\infty} d x f(x) e^{-i w x},
$$

are related by Fourier transform.

In order to apply this formula to (B.1a), we introduce the following notations

$$
x=q_{a} b^{a}, \quad y=q_{a} t^{a}, \quad \Theta=q_{a}\left(\zeta^{a}-b^{a} \zeta^{0}\right) .
$$

Then the function $f\left(x+q_{0}\right)$ entering the 1.h.s. of $(\overline{B .2})$ is found as

$$
f(x)=\sum_{m=1}^{\infty} \sum_{s= \pm 1} \frac{e^{-2 \pi \operatorname{ism}\left(\Theta+x \zeta_{0}\right)}}{(s m)^{\nu}} \int_{0}^{\infty} \frac{\mathrm{d} t}{t} \frac{t-\operatorname{sign}(x) \operatorname{siz}}{t+\operatorname{sign}(x) \operatorname{siz}} e^{-2 \pi m \operatorname{sign}(x) \mathcal{R}\left(\frac{x+\mathbf{i} y}{t}+t(x-\mathrm{i} y)\right)} .
$$

Its Fourier transform is obtained by using $\operatorname{sign}(x)$ to split the $x$-integration into two integrals along the half-axes. The resulting integrands are of the type $e^{-A x}, \operatorname{Re} A>0$. Their evaluation yields

$$
\tilde{f}(w)=\frac{1}{2 \pi} \sum_{m=1}^{\infty} \sum_{s, s^{\prime}= \pm 1} \frac{e^{-2 \pi \mathrm{i} s m \Theta}}{(s m)^{\nu}} \int_{0}^{\infty} \frac{\mathrm{d} t}{t} \frac{t-s s^{\prime} \mathbf{i z}}{t+s s^{\prime} \mathrm{i} \mathbf{z}} \frac{e^{-2 \pi \mathrm{i} m s^{\prime} \mathcal{R} y\left(t^{-1}-t\right)}}{m\left(\mathcal{R}\left(t^{-1}+t\right)+\mathrm{i} s s^{\prime} \zeta^{0}\right)+\frac{\mathrm{i} s^{\prime} w}{2 \pi}} .
$$

The sign variables $s$ and $s s^{\prime}$ can then be used to extend the sum over $m$ to negative values and the $t$-integral along the whole real axis, respectively. This should be contrasted with eq. (B.5) where the extension of the $t$-integral to the real axis cannot be performed. Substituting this result into the r.h.s. of (B.2) then yields

$$
\mathcal{I}_{\hat{\gamma}}^{(\nu)}=\frac{1}{2 \pi} \sum_{\substack{n \in \mathbb{Z} \\ m \neq 0}} \frac{e^{2 \pi \mathrm{i}(n x-m \Theta)}}{m^{\nu-1}|m|} \int_{-\infty}^{\infty} \frac{\mathrm{d} t}{t} \frac{t-\mathrm{i} \mathbf{z}}{t+\mathrm{i}} \frac{e^{-2 \pi \mathrm{i} m \mathcal{R} y\left(t^{-1}-t\right)}}{m\left(\mathcal{R}\left(t^{-1}+t\right)+\mathrm{i} \zeta^{0}\right)+\mathrm{i} n} .
$$

The $t$-integral can now be evaluated explicitly using the method of residues by closing the integration contour at infinity in the half-plane where the integrand is exponentially suppressed. The integrand has three poles located at $t=-\mathrm{i} \mathbf{z}$ and $t=-\mathrm{i} \mathbf{z}_{ \pm}^{m, n}$ with $\mathbf{z}_{ \pm}^{m, n}$ defined in (4.4). Their contribution depends on the signs

$$
\epsilon=\operatorname{sign} y, \quad \varepsilon=-\operatorname{sign}(\operatorname{Re} \mathbf{z}) .
$$


For $\epsilon= \pm 1$ the contour includes the poles at $\mathbf{z}_{ \pm}^{m, n}$, respectively. The pole at $t=$ -iz contributes for $m y \operatorname{Re} \mathbf{z}<0$ only, and yields different contributions on the four quadrants in the $(y, \operatorname{Re} \mathbf{z})$-plane. Introducing sign-functions in the appropriate places the resulting expressions can be combined to

$$
\mathcal{I}_{\hat{\gamma}}^{(\nu)}=\sum_{\substack{n \in \mathbb{Z} \\ m \neq 0}} \frac{e^{2 \pi i(n x-m \Theta)-2 \pi|y||m \tau+n|}}{m^{\nu}|m \tau+n|} \frac{\mathbf{z}_{\epsilon}^{m, n}+\mathbf{z}}{\mathbf{z}_{\epsilon}^{m, n}-\mathbf{z}}+2 \epsilon \sum_{\substack{n \in \mathbb{Z} \\ \epsilon \varepsilon m>0}} \frac{e^{2 \pi i(n x-m \Theta)}}{m^{\nu}} \frac{e^{2 \pi m y \mathcal{R}\left(\mathbf{z}^{-1}+\mathbf{z}\right)}}{m \xi^{0}+n} .
$$

This result completes the resummation of $\mathcal{I}_{\hat{\gamma}}^{(\nu)}$.

We now turn to the second "basic resummation" (B.1b). Substituting (A.8), it explicitly reads

$$
\mathcal{I}_{0}^{(2)}(\mathbf{z})=\sum_{q_{0} \neq 0} \sum_{m=1}^{\infty} \sum_{s= \pm 1} \frac{e^{-2 \pi \mathbf{i} s m q_{0} \zeta^{0}}}{m^{2}} \int_{0}^{\infty} \frac{\mathrm{d} t}{t} \frac{t-\operatorname{sign}\left(q_{0}\right) s \mathbf{i z}}{t+\operatorname{sign}\left(q_{0}\right) \operatorname{siz}} e^{-2 \pi m\left|q_{0}\right| \mathcal{R}\left(t^{-1}+t\right)} .
$$

The Poisson resummation formula then requires to include the $q_{0}=0$-term in the sum

$$
\mathcal{I}_{0}^{(2)}(\mathbf{z})=\sum_{q_{0} \in \mathbb{Z}} f\left(q_{0}\right)-f(0)
$$

For $q_{0}=0$, the $t$-integral arising from $(\mathrm{B} .10$ can be evaluated analytically. In fact, at every boundary it diverges logarithmically, but these divergences can be canceled by first combining contributions of $t$ and $1 / t$ since this transformation leaves the exponential invariant. Furthermore, the factors $\operatorname{sign}\left(q_{0}\right)$ can be removed by redefining $s \rightarrow \operatorname{sign}\left(q_{0}\right) s$, which leads to $\left|q_{0}\right|$ in the first exponential. The functions entering into (B.11) then read

$$
\begin{aligned}
f\left(q_{0}\right) & =\sum_{m=1}^{\infty} \sum_{s= \pm 1} \frac{e^{-2 \pi \mathrm{i} s m\left|q_{0}\right| \zeta^{0}}}{m^{2}} \int_{0}^{\infty} \frac{\mathrm{d} t}{t} \frac{t-s \mathrm{i} \mathbf{z}}{t+s i \mathbf{z}} e^{-2 \pi m\left|q_{0}\right| \mathcal{R}\left(t^{-1}+t\right)} \\
f(0) & =-\frac{2 \pi^{2}}{3} \log \mathbf{z} .
\end{aligned}
$$

Note that $f\left(q_{0}\right)$ is obtained from $(\mathbb{B} .5)$ by setting $q_{a}=0$. Its Poisson resummation can be obtained by following similar steps as for $\mathcal{I}_{\hat{\gamma}}^{(\nu)}$. The result turns out to be the same as (B.9) evaluated for $\nu=2, q_{a}=0$ and $\epsilon=1$, which can be verified by an explicit evaluation of the integrals. Substituting it into (B.11) then yields

$$
\mathcal{I}_{0}^{(2)}(\mathbf{z})=\sum_{\substack{n \in \mathbb{Z} \\ m \neq 0}} \frac{1}{m^{2}|m \tau+n|} \frac{\mathbf{z}_{+}^{m, n}+\mathbf{z}}{\mathbf{z}_{+}^{m, n}-\mathbf{z}}+\sum_{\substack{n \in \mathbb{Z} \\ m>0}} \frac{2 \varepsilon}{m^{2}\left(m \xi^{0}+n\right)}+\frac{2 \pi^{2}}{3} \log \mathbf{z} .
$$

Notice that the second sum over $n$ converges because the combination of $n$th and $-n$th terms scales like $n^{-2}$. This result concludes the Poisson resummation of (B.1) and we will now proceed with its application to the Type IIA twistor lines. 


\section{B.2 Resuming the Type IIA twistor lines}

Under the decomposition $\gamma=\left\{q_{0}, \hat{\gamma}\right\}$ the instanton corrections to the twistor lines (A.7) give rise to four types of terms: $q_{a} \mathcal{I}_{\hat{\gamma}}^{(1)}$ linear in charges, $\mathcal{I}_{\hat{\gamma}}^{(2)}$ without charges, $q_{0} \mathcal{I}_{\gamma}^{(1)}$ linear in $q_{0}$, and terms with $\mathcal{I}_{0}^{(2)}$. By virtue of the relation $\mathcal{K}_{\gamma}=\frac{\mathrm{i}}{4} \mathcal{I}_{\gamma}^{(1)}(0)$, the contributions of the form $q_{a} \mathcal{K}_{\gamma}$ and $q_{0} \mathcal{K}_{\gamma}$ result as a special case.

Taking into account the sum over charges and that $n_{\gamma}=n_{\hat{\gamma}}=n_{-\hat{\gamma}}$ for $\hat{\gamma} \neq 0$, the expressions for the first two types of terms can be summarized as

$$
\begin{aligned}
\sum_{\hat{\gamma} \neq 0} n_{\hat{\gamma}} q_{a}^{2-\nu} \mathcal{I}_{\hat{\gamma}}^{(\nu)}=2 \sum_{\hat{\gamma}_{+}} n_{\hat{\gamma}} q_{a}^{2-\nu} & {\left[\sum_{\substack{n \in \mathbb{Z} \\
m \neq 0}} \frac{\mathrm{e}^{2 \pi i(n x-m \Theta)}}{m^{\nu}} \frac{\mathrm{e}^{-2 \pi y|m \tau+n|}}{|m \tau+n|} \frac{\mathbf{z}_{+}^{m, n}+\mathbf{z}}{\mathbf{z}_{+}^{m, n}-\mathbf{z}}\right.} \\
+ & \left.2 \varepsilon^{\nu+1} \sum_{\substack{n \in \mathbb{Z} \\
m>0}} \frac{\mathrm{e}^{2 \pi \varepsilon\left(m y \mathcal{R}\left(\mathbf{z}^{-1}+\mathbf{z}\right)+\mathrm{i}(n x-m \Theta)\right)}}{m^{\nu}\left(m \xi^{0}+n\right)}\right] .
\end{aligned}
$$

Here $\hat{\gamma}_{+}$is the set of charges satisfying $y>0$ and already appeared in the description of Section 6 . The terms linear in $q_{0}$ are related to this result by

$$
\sum_{q_{0} \in \mathbb{Z}} q_{0} \mathcal{I}_{\gamma}^{(\nu)}(\mathbf{z})=-\frac{1}{2 \pi \mathrm{i}} \partial_{\zeta^{0}} \mathcal{I}_{\hat{\gamma}}^{(\nu+1)}
$$

which can be established based on the definition (A.8) where the r.h.s. is expressed in terms of Type IIA variables. Finally, either setting $\mathbf{z}=0$ in the above equations or redoing calculations from the very beginning, one can show that

$$
\begin{aligned}
& \sum_{\hat{\gamma} \neq 0} n_{\hat{\gamma}} q_{a} \sum_{q_{0} \in \mathbb{Z}} \mathcal{K}_{\gamma}=\frac{\mathrm{i}}{2} \sum_{\hat{\gamma}_{+}} n_{\hat{\gamma}} q_{a} \sum_{\substack{n \in \mathbb{Z} \\
m \neq 0}} \frac{e^{2 \pi i(n x-m \Theta)-2 \pi|y||m \tau+n|}}{m|m \tau+n|}, \\
& \sum_{\gamma} n_{\gamma} q_{0} \mathcal{K}_{\gamma}=-\frac{1}{4 \pi} \sum_{\substack{n \in \mathbb{Z} \\
m \neq 0}}\left[\sum_{\hat{\gamma}_{+}} n_{\hat{\gamma}} \partial_{\zeta^{0}} \frac{e^{2 \pi i(n x-m \Theta)-2 \pi y|m \tau+n|}}{m^{2}|m \tau+n|}+\frac{\chi_{Y}}{2} \frac{m \tau_{1}+n}{m|m \tau+n|^{3}}\right] .
\end{aligned}
$$

With these relations, we now have all the ingredients to compute the resummed Type IIA twistor lines (A.7) and compare them to their Type IIB counterparts (4.7). Since one starts from the Type IIA side, we work in the patch $\mathcal{U}_{0_{A}}$. The decomposition of the sum over charges in $\tilde{\xi}_{\Lambda}^{\left[0_{A}\right]}$ then yields

$$
\begin{aligned}
& \tilde{\xi}_{a}^{\left[0_{A}\right]}=\frac{\mathrm{i}}{2}\left(\tilde{\zeta}_{a}+\mathcal{R}\left(\mathbf{z}^{-1} F_{a}-\mathbf{z} \bar{F}_{a}\right)\right)+\frac{\mathrm{i}}{32 \pi^{2}} \sum_{\hat{\gamma} \neq 0} n_{\hat{\gamma}} q_{a} \mathcal{I}_{\hat{\gamma}}^{(1)}, \\
& \tilde{\xi}_{0}^{\left[0_{A}\right]}=\frac{\mathrm{i}}{2}\left(\tilde{\zeta}_{0}+\mathcal{R}\left(\mathbf{z}^{-1} F_{0}-\mathbf{z} \bar{F}_{0}\right)\right)-\frac{1}{64 \pi^{3}} \partial_{\zeta^{0}}\left(\sum_{\hat{\gamma} \neq 0} n_{\hat{\gamma}} \mathcal{I}_{\hat{\gamma}}^{(2)}-\chi_{Y} \mathcal{I}_{0}^{(2)}\right) .
\end{aligned}
$$


In order to facilitate the comparison between the last twistor lines, it turns out to be convenient to trade $\alpha_{\mathrm{A}}^{\left[0_{A}\right]}$ for the combination $\alpha_{\mathrm{B}}^{\left[0_{A}\right]}$ given in (3.12). For the latter quantity the decomposition gives

$$
\begin{aligned}
\alpha_{\mathrm{B}}^{\left[0_{A}\right]}=- & \frac{\mathrm{i}}{4}\left[\sigma+\mathcal{R}\left(\mathbf{z}^{-1} W-\mathbf{z} \bar{W}\right)\right. \\
& \left.\quad-\left(\zeta^{\Lambda}+\mathcal{R}\left(\mathbf{z}^{-1} z^{\Lambda}-\mathbf{z} \bar{z}^{\Lambda}\right)\right)\left(\tilde{\zeta}_{\Lambda}+\mathcal{R}\left(\mathbf{z}^{-1} F_{\Lambda}-\mathbf{z} \bar{F}_{\Lambda}\right)\right)\right] \\
- & \frac{\chi_{Y}}{96 \pi} \log \mathbf{z}-\frac{1}{64 \pi^{3}}\left[\sum_{\hat{\gamma} \neq 0} n_{\hat{\gamma}} \mathcal{I}_{\hat{\gamma}}^{(2)}-\chi_{Y} \mathcal{I}_{0}^{(2)}\right] \\
+ & \frac{\mathcal{R}}{8 \pi^{2}}\left[\sum_{\hat{\gamma} \neq 0} n_{\hat{\gamma}} q_{a}\left(\mathbf{z}^{-1} z^{a}+\mathbf{z} \bar{z}^{a}\right) \sum_{q_{0} \in \mathbb{Z}} \mathcal{K}_{\gamma}+\left(\mathbf{z}^{-1}+\mathbf{z}\right) \sum_{\gamma} n_{\gamma} q_{0} \mathcal{K}_{\gamma}\right] .
\end{aligned}
$$

Substituting the relations $(\mathbb{B} .13)$ - (B.16), a straightforward though somewhat tedious computation allows to establish the following relation between the Type IIA and Type IIB twistor lines

$$
\tilde{\xi}_{\Lambda}^{\left[0_{A}\right]}=\tilde{\xi}_{\Lambda}^{\left[0_{B}\right]}+\tilde{\mathcal{T}}_{\Lambda}, \quad \alpha_{\mathrm{B}}^{\left[0_{A}\right]}=\alpha_{\mathrm{B}}^{\left[0_{B}\right]}+\mathcal{T}_{\alpha},
$$

where we introduced

$$
\begin{aligned}
& \tilde{\mathcal{T}}_{a}=\frac{\mathrm{i}}{8 \pi^{2}} \sum_{q_{a} \geq 0} n_{q_{a}}^{(0)} q_{a} \sum_{\substack{n \in \mathbb{Z} \\
m>0}} \frac{\mathrm{e}^{-2 \pi \mathrm{i} \varepsilon q_{a}\left(m \xi^{a}-b^{a}\left(m \xi^{0}+n\right)\right)}}{m\left(m \xi^{0}+n\right)} \\
& \tilde{\mathcal{T}}_{0}=\frac{1}{16 \pi^{3}} \sum_{q_{a} \geq 0} n_{q_{a}}^{(0)} \sum_{\substack{n \in \mathbb{Z} \\
m>0}}\left(\varepsilon-2 \pi \mathrm{i} q_{a} b^{a}\left(m \xi^{0}+n\right)\right) \frac{\mathrm{e}^{-2 \pi \mathrm{i} \varepsilon q_{a}\left(m \xi^{a}-b^{a}\left(m \xi^{0}+n\right)\right)}}{m\left(m \xi^{0}+n\right)^{2}} \\
& \mathcal{T}_{\alpha}=-\frac{\varepsilon}{16 \pi^{3}} \sum_{q_{a} \geq 0} n_{q_{a}}^{(0)} \sum_{\substack{n \in \mathbb{Z} \\
m>0}} \frac{\mathrm{e}^{-2 \pi \mathrm{i} \varepsilon q_{a}\left(m \xi^{a}-b^{a}\left(m \xi^{0}+n\right)\right)}}{m^{2}\left(m \xi^{0}+n\right)}
\end{aligned}
$$

In order to verify these identities, it is useful to note that the terms proportional to $\chi_{Y}$ provide the $q_{a}=0$ part of the sums over charges once (A.2) is applied. Furthermore, we have

$$
\begin{aligned}
2 \pi y|m \tau+n|-2 \pi \mathrm{i}(n x-m \Theta) & =S_{m, n, q_{a}}, \\
-m y \mathcal{R}\left(\mathbf{z}^{-1}+\mathbf{z}\right)-\mathrm{i}(n x-m \Theta) & =\mathrm{i} q_{a}\left(m \xi^{a}-b^{a}\left(m \xi^{0}+n\right)\right) .
\end{aligned}
$$

Tracing back the origin of the terms appearing in $(\mathbb{B} .19)$, it is worthwhile noting that the twistor lines $\tilde{\xi}_{\Lambda}^{\left[0_{B}\right]}, \alpha_{\mathrm{B}}^{\left[0_{B}\right]}$ are generated by the poles $t=-\mathrm{i} \mathbf{z}_{ \pm}^{m, n}$ while the $\mathcal{T}$-terms originate from the pole at $t=-\mathrm{iz}$.

In fact, it can be demonstrated that away from the real and imaginary axes, the additional contributions $\tilde{\mathcal{T}}_{\Lambda}, \mathcal{T}_{\alpha}$ are holomorphic functions of $\xi^{\Lambda}$. This is a crucial prerequisite for removing these terms by an appropriate gauge transformation. To show 
this, one should "undo" the Poisson resummation on $n$ for these extra contributions. In this course, we need the following relations in the sector $q_{a} \neq 0$

$$
\begin{aligned}
& \sum_{n \in \mathbb{Z}} \frac{\mathrm{e}^{2 \pi \mathrm{i} \varepsilon q_{a} b^{a}\left(m \xi^{0}+n\right)}}{m \xi^{0}+n}=2 \pi \mathrm{i} \varepsilon \sum_{q_{0} \in \mathbb{Z}} \Delta_{\gamma}(\mathbf{z}) \mathrm{e}^{-2 \pi \mathrm{i} \varepsilon m q_{0} \xi^{0}}, \\
& \sum_{n \in \mathbb{Z}}\left(1-2 \pi \mathrm{i} \varepsilon q_{a} b^{a}\left(m \xi^{0}+n\right)\right) \frac{\mathrm{e}^{2 \pi \mathrm{i} \varepsilon q_{a} b^{a}\left(m \xi^{0}+n\right)}}{\left(m \xi^{0}+n\right)^{2}}=-4 \pi^{2} \sum_{q_{0} \in \mathbb{Z}} q_{0} \Delta_{\gamma}(\mathbf{z}) \mathrm{e}^{-2 \pi \mathrm{i} \varepsilon m q_{0} \xi^{0}},
\end{aligned}
$$

where we introduced the step-function

$$
\Delta_{\gamma}(\mathbf{z})= \begin{cases}0 & \text { if } \quad \epsilon_{\gamma} \operatorname{Re} \mathbf{z} \operatorname{Im} \mathbf{z}>0 \quad \Leftrightarrow \quad \epsilon_{\gamma} \varepsilon \tilde{\varepsilon}=-1 \\ \epsilon_{\gamma} & \text { if } \quad \epsilon_{\gamma} \operatorname{Re} \mathbf{z} \operatorname{Im} \mathbf{z}<0 \quad \Leftrightarrow \quad \epsilon_{\gamma} \varepsilon \tilde{\varepsilon}=1\end{cases}
$$

and $\tilde{\varepsilon}=\operatorname{sign}(\operatorname{Im} \mathbf{z})$. These relations can be obtained in the usual way by performing Poisson resummation which boils down to the evaluation of continuous Fourier transform w.r.t. the variable $n$. The latter is found by closing the integration contour in the upper (lower) half-plane where the integrand is exponentially suppressed. Taking into account that $m>0$, the analysis of the pole-structure reveals that the integral is non-zero for $\epsilon_{\gamma} \varepsilon \tilde{\varepsilon}=1$ and vanishes otherwise, which leads to the appearance of the step-function. In the sector $q_{a}=0$, a similar resummation gives

$$
\begin{gathered}
\sum_{n \in \mathbb{Z}} \frac{1}{m \xi^{0}+n}=\sum_{n \in \mathbb{Z}} \frac{m \xi^{0}}{\left(m \xi^{0}\right)^{2}-n^{2}}=2 \pi \mathrm{i} \tilde{\varepsilon}\left(\sum_{q_{0}=1}^{\infty} \mathrm{e}^{-2 \pi \mathrm{i} \tilde{\varepsilon} m q_{0} \xi^{0}}+1 / 2\right), \\
\sum_{n \in \mathbb{Z}} \frac{1}{\left(m \xi^{0}+n\right)^{2}}=\sum_{n \in \mathbb{Z}} \frac{\left(m \xi^{0}\right)^{2}+n^{2}}{\left(\left(m \xi^{0}\right)^{2}-n^{2}\right)^{2}}=-4 \pi^{2} \sum_{q_{0}=1}^{\infty} q_{0} \mathrm{e}^{-2 \pi \mathrm{i} \tilde{\varepsilon} m q_{0} \xi^{0}} .
\end{gathered}
$$

Substituting the identities (B.22), (B.24) into eqs. (B.20) and carrying out the summation over $m$ explicitly then leads to our final result

$$
\begin{aligned}
& \tilde{\mathcal{T}}_{a}=\frac{\varepsilon}{4 \pi} \sum_{q_{0} \in \mathbb{Z}, q_{a} \geq 0} n_{q_{a}}^{(0)} q_{a} \Delta_{\gamma}(\mathbf{z}) \log \left(1-\mathrm{e}^{-2 \pi \mathrm{i} \varepsilon q_{\Lambda} \xi^{\Lambda}}\right), \\
& \tilde{\mathcal{T}}_{0}=\frac{\varepsilon}{4 \pi} \sum_{q_{0} \in \mathbb{Z}, q_{a} \geq 0} n_{q_{a}}^{(0)} q_{0} \Delta_{\gamma}(\mathbf{z}) \log \left(1-\mathrm{e}^{-2 \pi \mathrm{i} \varepsilon q_{\Lambda} \xi^{\Lambda}}\right), \\
& \mathcal{T}_{\alpha}=-\frac{\mathrm{i}}{8 \pi^{2}} \sum_{\substack{q_{0} \in \mathbb{Z}, q_{a} \geq 0 \\
\gamma \neq 0}} n_{q_{a}}^{(0)} \Delta_{\gamma}(\mathbf{z}) \operatorname{Li}_{2}\left(\mathrm{e}^{-2 \pi \mathrm{i} \varepsilon q_{\Lambda} \xi^{\Lambda}}\right)-\frac{\mathrm{i} \varepsilon \tilde{\varepsilon}}{192} \chi_{X} .
\end{aligned}
$$

In the regions where the step-function $\Delta_{\gamma}$ is constant, the $\mathcal{T}$-contributions indeed depend only on the twistor lines $\xi^{\Lambda}$ in a holomorphic way. Moreover, it is straightforward to see that, away from the discontinuities induced by $\varepsilon$ and $\tilde{\varepsilon}, \tilde{\mathcal{T}}_{\Lambda}=\partial_{\xi^{\Lambda}} \mathcal{T}_{\alpha}$, which is a necessary requirement for the extra terms to constitute a gauge transformation. 


\section{SL(2, Z)-transformation of the Type IIB twistor lines}

The key ingredient in the construction of the non-perturbative mirror map (5.1) is the fact that the $\operatorname{SL}(2, \mathbb{Z})$-transformation of the Type IIB twistor lines $(5.3)$ - (5.5) is given by the classical transformation law (3.13). In this appendix we provide the details underlying the derivation of this result.

First, it is useful to decompose the transformation of $\tau=\tau_{1}+\mathrm{i} \tau_{2}$, eq. (3.9), into its real and imaginary part

$$
\tau_{2} \mapsto \frac{\tau_{2}}{|c \tau+d|^{2}}, \quad \tau_{1} \mapsto \frac{a c|\tau|^{2}+b d+(a d+b c) \tau_{1}}{|c \tau+d|^{2}} .
$$

Moreover, using the notation $\mathbf{z}_{ \pm}^{c, d}$ introduced in (4.4), the transformation of $\mathbf{z}$ (3.14) can be expressed as

$$
\mathbf{z} \mapsto \frac{1+\mathbf{z}_{-}^{c, d} \mathbf{z}}{\mathbf{z}_{-}^{c, d}-\mathbf{z}}=-\frac{\mathbf{z}_{+}^{c, d}-\mathbf{z}}{1+\mathbf{z}_{+}^{c, d} \mathbf{z}}
$$

which implies

$$
\mathbf{z}^{-1}+\mathbf{z} \mapsto \frac{|c \tau+d|}{c \xi^{0}+d}\left(\mathbf{z}^{-1}+\mathbf{z}\right), \quad \mathbf{z}^{-1}-\mathbf{z} \mapsto \frac{\left(c \tau_{1}+d\right)\left(\mathbf{z}^{-1}-\mathbf{z}\right)-2 c \tau_{2}}{c \xi^{0}+d}
$$

With regards to the transformation of the instanton-sums appearing in the twistor lines, it is useful to introduce

$$
\left(\begin{array}{l}
m^{\prime} \\
n^{\prime}
\end{array}\right)=\left(\begin{array}{ll}
a & c \\
b & d
\end{array}\right)\left(\begin{array}{l}
m \\
n
\end{array}\right)
$$

Utilizing this notation, one then proves the transformation properties

$$
S_{m, n, q_{a}} \mapsto S_{m^{\prime}, n^{\prime}, q_{a}}, \quad|m \tau+n| \mapsto \frac{\left|m^{\prime} \tau+n^{\prime}\right|}{|c \tau+d|}
$$

which enter into all twistor lines. With these preliminary results, we are now in the position to discuss the transformation of $\tilde{\xi}_{a}, \tilde{\xi}_{0}$, and $\alpha_{\mathrm{B}}$ in turn.

We start by considering $\tilde{\xi}_{a}$. Noting that the second term appearing in (3.13) is already generated by the classical piece in the first line of eq. (5.3), it follows that the instanton contribution in the second line must be modular invariant. That this is indeed the case readily follows from (C.5) together with the invariance of

$$
\frac{1+\mathbf{z}_{+}^{m, n} \mathbf{z}}{\mathbf{z}-\mathbf{z}_{+}^{m, n}} \mapsto \frac{1+\mathbf{z}_{+}^{m^{\prime}, n^{\prime}} \mathbf{z}}{\mathbf{z}-\mathbf{z}_{+}^{m^{\prime}, n^{\prime}}}
$$


Concerning the transformation of $\tilde{\xi}_{0}$, we first verify that the classical pieces given by the first line of (5.4) satisfies the classical (3.13). Hence the transformation of the instanton contributions given by the second and third line has to reproduce the instanton pieces originating from $\left(c \xi^{0}+d\right) \tilde{\xi}_{0}-c\left(\alpha_{\mathrm{B}}-\xi^{a} \tilde{\xi}_{a}\right)$. This can be verified by applying the identities

$$
\begin{gathered}
\frac{m \tau_{1}+n}{|m \tau+n|^{2}} \mapsto \frac{d\left(m^{\prime} \tau_{1}+n^{\prime}\right)+c\left(n^{\prime} \tau_{1}+m^{\prime}|\tau|^{2}\right)}{\left|m^{\prime} \tau+n^{\prime}\right|^{2}}, \quad m \xi^{0}+n \mapsto \frac{m^{\prime} \xi^{0}+n^{\prime}}{c \xi^{0}+d}, \\
\frac{1-\mathbf{z}_{+}^{m, n} \mathbf{z}}{\mathbf{z}-\mathbf{z}_{+}^{m, n}} t^{a} \mapsto\left(c \tau_{1}+d\right) \frac{1-\mathbf{z}_{+}^{m^{\prime}, n^{\prime}} \mathbf{z}}{\mathbf{z}-\mathbf{z}_{+}^{m^{\prime}, n^{\prime}}} t^{a}-c \frac{\mathbf{z}+\mathbf{z}_{+}^{m^{\prime}, n^{\prime}}}{\mathbf{z}-\mathbf{z}_{+}^{m^{\prime}, n^{\prime}}} \tau_{2} t^{a}
\end{gathered}
$$

Finally, following the same strategy as for $\tilde{\xi}_{0}$, on verifies the transformation law of $\alpha_{\mathrm{B}}$ by first establishing the intermediate result

$$
\left(m \tau_{1}+n\right)\left(\mathbf{z}^{-1}-\mathbf{z}\right)-2 m \tau_{2} \mapsto \frac{\left(m^{\prime} \tau_{1}+n^{\prime}\right)\left(\mathbf{z}^{-1}-\mathbf{z}\right)-2 m^{\prime} \tau_{2}}{c \xi^{0}+d}
$$

This completes the proof that the classical $\mathrm{SL}(2, \mathbb{Z})$ transformations (3.13) do not receive quantum corrections from $\mathrm{D}(-1)$ and D1-brane instantons.

\section{References}

[1] H. Ooguri and C. Vafa, "Summing up D-instantons," Phys. Rev. Lett. 77 (1996) 3296-3298, hep-th/9608079.

[2] K. Becker, M. Becker, and A. Strominger, "Five-branes, membranes and nonperturbative string theory," Nucl. Phys. B456 (1995) 130-152, hep-th/9507158.

[3] S. Cecotti, S. Ferrara, and L. Girardello, "Geometry of type II superstrings and the moduli of superconformal field theories," Int. J. Mod. Phys. A4 (1989) 2475.

[4] S. Ferrara and S. Sabharwal, "Quaternionic manifolds for type II superstring vacua of Calabi-Yau spaces," Nucl. Phys. B332 (1990) 317.

[5] K. Hori et al., "Mirror symmetry,". Providence, USA: AMS (2003) 929 p.

[6] P. Candelas, X. C. de la Ossa, P. S. Green, and L. Parkes, "A pair of Calabi-Yau manifolds as an exactly soluble superconformal theory," Nucl. Phys. B359 (1991) $21-74$.

[7] S. Ferrara, J. A. Harvey, A. Strominger, and C. Vafa, "Second quantized mirror symmetry," Phys. Lett. B361 (1995) 59-65, hep-th/9505162.

[8] P. S. Aspinwall, "D-branes on Calabi-Yau manifolds," hep-th/0403166. 
[9] M. Kontsevich, "Homological algebra of mirror symmetry," in Proceedings of the International Congress of Mathematicians, Vol. 1, 2 (Zürich, 1994), pp. 120-139. Birkhäuser, Basel, 1995. alg-geom/9411018.

[10] R. Böhm, H. Günther, C. Herrmann, and J. Louis, "Compactification of type IIB string theory on Calabi-Yau threefolds," Nucl. Phys. B569 (2000) 229-246, hep-th/9908007.

[11] H. Ooguri, Y. Oz, and Z. Yin, "D-branes on Calabi-Yau spaces and their mirrors," Nucl. Phys. B477 (1996) 407-430, hep-th/9606112.

[12] D. Robles-Llana, F. Saueressig, U. Theis, and S. Vandoren, "Membrane instantons from mirror symmetry," arXiv:0707.0838 [hep-th].

[13] F. Saueressig, "Recent results in four-dimensional non-perturbative string theory," $J$. Phys. Conf. Ser. 110 (2008) 102010, arXiv:0710.4931.

[14] B. de Wit, "N = 2 electric-magnetic duality in a chiral background," Nucl. Phys. Proc. Suppl. 49 (1996) 191-200, hep-th/9602060.

[15] J. Manschot, "Stability and duality in N=2 supergravity," arXiv:0906.1767.

[16] D. Robles-Llana, M. Roček, F. Saueressig, U. Theis, and S. Vandoren, "Nonperturbative corrections to 4D string theory effective actions from $\mathrm{SL}(2, \mathrm{Z})$ duality and supersymmetry," Phys. Rev. Lett. 98 (2007) 211602, hep-th/0612027.

[17] A. Collinucci, P. Soler, and A. M. Uranga, "Non-perturbative effects and wall-crossing from topological strings," arXiv:0904.1133.

[18] F. Saueressig and S. Vandoren, "Conifold singularities, resumming instantons and nonperturbative mirror symmetry," JHEP 07 (2007) 018, arXiv:0704.2229 [hep-th].

[19] S. Alexandrov, B. Pioline, F. Saueressig, and S. Vandoren, "D-instantons and twistors," JHEP 03 (2009) 044, arXiv:0812.4219.

[20] S. Alexandrov, "D-instantons and twistors: some exact results," arXiv:0902.2761.

[21] S. Alexandrov, F. Saueressig, and S. Vandoren, "Membrane and fivebrane instantons from quaternionic geometry," JHEP 09 (2006) 040, hep-th/0606259.

[22] B. Pioline, "The automorphic NS5-brane," arXiv:0902.3274.

[23] S. Alexandrov, B. Pioline, F. Saueressig, and S. Vandoren, "Linear perturbations of Hyperkähler metrics," arXiv:0806.4620 [hep-th].

[24] S. Alexandrov, B. Pioline, F. Saueressig, and S. Vandoren, "Linear perturbations of quaternionic metrics.," arXiv:0810.1675. 
[25] D. Robles-Llana, F. Saueressig, and S. Vandoren, "String loop corrected hypermultiplet moduli spaces," JHEP 03 (2006) 081, hep-th/0602164.

[26] S. Alexandrov, "Quantum covariant c-map," JHEP 05 (2007) 094, hep-th/0702203.

[27] S. M. Salamon, "Differential geometry of quaternionic manifolds," Annales Scientifiques de l'École Normale Supérieure (4) 19 (1986), no. 1, 31-55.

[28] C. LeBrun, "Quaternionic-Kähler manifolds and conformal geometry," Math. Ann. 284 (1989), no. 3, 353-376.

[29] H. Geiges, "Contact geometry," in Handbook of differential geometry. Vol. II, pp. 315-382. Elsevier/North-Holland, Amsterdam, 2006. math/0307242.

[30] K. Galicki, "A generalization of the momentum mapping construction for quaternionic Kähler manifolds," Comm. Math. Phys. 108 (1987), no. 1, 117-138.

[31] M. Bodner, A. C. Cadavid, and S. Ferrara, "(2,2) vacuum configurations for type IIA superstrings: $\mathrm{N}=2$ supergravity Lagrangians and algebraic geometry," Class. Quant. Grav. 8 (1991) 789-808.

[32] I. Antoniadis, S. Ferrara, R. Minasian, and K. S. Narain, " $R^{4}$ couplings in M- and type II theories on Calabi-Yau spaces," Nucl. Phys. B507 (1997) 571-588, hep-th/9707013.

[33] H. Günther, C. Herrmann, and J. Louis, "Quantum corrections in the hypermultiplet moduli space," Fortsch. Phys. 48 (2000) 119-123, hep-th/9901137.

[34] A. Neitzke, B. Pioline, and S. Vandoren, "Twistors and Black Holes," JHEP 04 (2007) 038, hep-th/0701214.

[35] B. de Wit and F. Saueressig, "Off-shell $\mathcal{N}=2$ tensor supermultiplets," JHEP 09 (2006) 062, hep-th/0606148.

[36] M. Davidse, F. Saueressig, U. Theis, and S. Vandoren, "Membrane instantons and de Sitter vacua," JHEP 09 (2005) 065, hep-th/0506097.

[37] B. Pioline and S. Vandoren, "Large D-instanton effects in string theory," arXiv:0904.2303. 